\title{
ON A RELATIVE FOURIER-MUKAI TRANSFORM ON GENUS ONE FIBRATIONS
}

\author{
IGOR BURBAN AND BERND KREUSSLER
}

\begin{abstract}
We study relative Fourier-Mukai transforms on genus one fibrations with section, allowing explicitly the total space of the fibration to be singular and non-projective. Grothendieck duality is used to prove a skew-commutativity relation between this equivalence of categories and certain duality functors. We use our results to explicitly construct examples of semi-stable sheaves on degenerating families of elliptic curves.
\end{abstract}

\section{INTRODUCTION}

Mukai [25] introduced functors of the form $\boldsymbol{R} \pi_{2 *}\left(\mathcal{P} \stackrel{L}{\otimes} \pi_{1}^{*}(\cdot)\right)$ as an efficient tool to study vector bundles on Abelian varieties. If such a functor is an equivalence of derived categories, it is called a FourierMukai transform, whereas in general they are referred to as integral transforms. Originally, they have been applied to study moduli problems on smooth projective varieties, not only on complex tori. This seems to be natural in the light of Orlov's theorem [26, which states that any auto-equivalence of the bounded derived category of coherent sheaves on a smooth projective variety $X$ is a Fourier-Mukai transform.

More recently, in higher dimensional birational geometry, the point of view was adopted that bounded derived categories of coherent sheaves might provide the framework which is needed to understand the minimal model programme. This is supported by results which show that, if two threefolds are related by a flop, their derived categories of bounded complexes of coherent sheaves are equivalent, see [9, 14, 22].

The minimal model programme naturally leads to the study of singular projective varieties. With the recent development in mind, this generates a demand for the study of Fourier-Mukai transforms on singular varieties. However, not only Orlov's representability theorem, but most of the results concerning equivalences between derived categories of coherent sheaves are established in the smooth case only, see [26]. The smoothness assumption enters the proofs in an essential way

2000 Mathematics Subject Classification. 18E30, 14H60, 14H20, 14J27, 14H10. 
by using that any coherent sheaf has a finite locally free resolution and a finite quasi-coherent injective resolution. Consequently, all the standard functors of Grothendieck are defined on and take values in the bounded derived category of coherent sheaves. This is no longer true on a singular variety. To deal with difficulties like these, we apply the machinery which was developed in [20].

Using derived categories and Fourier-Mukai transforms, Chen [14] studied flops on three-dimensional projective varieties with terminal Gorenstein singularities. Using non-commutative algebra, similar results were proved in a more general setting by Van den Bergh [28]. Some general properties of integral transforms on singular varieties can be found in Chen's paper, but the main difficulties with singularities are circumvented by embedding such a variety into a smooth fourdimensional variety. Another method to circumvent these difficulties was used by Kawamata 22].

On elliptically fibred smooth projective varieties, relative FourierMukai transforms have been applied successfully to the study of moduli of vector bundles, see [7, 10, 30. Caldararu [13] studied relative Fourier-Mukai transforms on smooth elliptic threefolds and is forced to use twisted sheaves, because he does not suppose the existence of a section. Relative Fourier-Mukai transforms on elliptic fibrations are now established as important tools in other areas as well, such as string theory, where D-branes are studied [3, 16] and also in the study of Calogero-Moser systems [5].

In this paper, we consider elliptic fibrations $q: X \rightarrow S$ with irreducible fibres and with a section. We do not need to suppose $X$ or $S$ to be either projective or smooth. Under these assumptions, we show in Theorem 2.12 that a particular integral transform is an autoequivalence of derived categories of bounded (resp. bounded above) complexes of coherent sheaves on $X$. This generalises 4, Theorem 2.8, [7], Theorem 5.3 and [10], Theorem 1.2.

Because we allow $X$ to be singular, the requirement that the fibres are irreducible is not too restrictive, at least in the two dimensional case. Namely, if $X \rightarrow S$ is a smooth elliptic surface with a section, it follows from Kodaira's classification of singular elliptic fibres that the components of a singular fibre, which are not met by the section, always form a negative definite configuration. In fact, such a configuration can be contracted to a rational double point.

Motivated by possible applications to mirror symmetry and integrable systems, we look at this situation from the point of view of the study of the degeneration of derived categories in families of smooth elliptic curves. If the elliptic curve degenerates to a singular irreducible 
curve, the total space may be singular. Therefore, it is important to allow the total space $X$ to be singular. In the singular case, however, it is difficult to prove that a given integral transform is an equivalence of categories, because the methods of Bridgeland [8] and Bondal, Orlov 6] do not apply. We overcome such difficulties by using a completely different strategy which allows us to use results from our earlier paper 12, in which the special case $S=\operatorname{Spec}(\boldsymbol{k})$ was studied.

The plan of this article is the following. We start Section 2 with recalling basic properties of the compactified relative Jacobian, following Altman and Kleiman 1, 2. Instead of using a Poincaré sheaf on the fibred product of the fibration $q: X \rightarrow S$ with its dual fibration, we prefer to work on $X \times_{S} X$ and give an explicit description of the sheaf $\mathcal{P}$ which defines the integral transform. Of course, these two approaches are equivalent. We give detailed proofs of some important properties of $\mathcal{P}$, versions of which can also be found in 4]. After proving a compatibility property between relative Fourier-Mukai transforms and direct image functors of closed embeddings, we show our main result in this section, Theorem 2.12, which states that the relative Fourier-Mukai transform with kernel $\mathcal{P}$ is an auto-equivalence of the derived category of coherent sheaves on the total space $X$.

In Section 3 we derive a certain skew-commutativity of the relative Fourier-Mukai transform $\operatorname{FM}_{\mathcal{P}}$ and the derived functor $\boldsymbol{R \mathcal { H } o m}(\cdot, \mathcal{L})$, where $\mathcal{L}$ is a line bundle on $X$. If $X$ is Gorenstein, this functor is a dualising functor on $X$ and we obtained a generalisation of Mukai's result [25], (3.8) as well as a more general form of our result [12], Theorem 6.11.

In the final Section 4, we give two examples of flat families of coherent sheaves on a singular fibration of cubics. In both examples, the general fibre of the family is a semi-stable vector bundle on a smooth elliptic curve. The degeneration on the singular fibre has an interesting semistable direct summand. In one example it is a torsion free but not locally free sheaf, whereas in the other example it is a vector bundle which is not a twist of an Atiyah bundle.

Acknowledgement. Both authors would like to thank U. Bruzzo, D. Hernández Ruipérez, B. Keller, M. Lehn and P. Schapira for fruitful discussions. The first-named author would like to thank R. Rouquier and the Institut de Mathématiques de Chevaleret in Paris for an invitation and constant support. The main work on this article has been done during a visit of the second-named author at the Institut de Mathématiques de Chevaleret, Paris, which was made possible through Research Seed Funding at Mary Immaculate College. Both authors are 
grateful to the Mathematisches Forschungsinstitut Oberwolfach, where they could carry out part of the work on this article.

Notation. We fix an algebraically closed field $\boldsymbol{k}$ of characteristic zero. Throughout this paper we work in the category of $\boldsymbol{k}$-schemes. Unless otherwise stated, a point is always a closed point and a fibre means a fibre over a closed point. If $y \in Y$ is a point, we denote by $\boldsymbol{k}(y)$ the residue field of $y$ and consider it as a sheaf with support at $y$. A morphism of schemes $f: Y \rightarrow T$ is said to have pure dimension $n$, if $\operatorname{dim} \mathcal{O}_{Y_{t}, y}=n$ for all points $y \in Y$, where $t=f(y)$ and $Y_{t}$ denotes the fibre of $f$ over $t \in T$. If $Y$ and $T$ are locally of finite type over $\boldsymbol{k}$, any morphism $f: Y \rightarrow T$ is locally of finite type. Such a morphism is called Cohen-Macaulay (resp. Gorenstein), if it is flat and all fibres are Cohen-Macaulay (resp. Gorenstein) schemes.

If $X$ is a separated Noetherian scheme of finite dimension, we denote by $\mathrm{D}_{\text {coh }}(X)$ the derived category of the category of complexes of $\mathcal{O}_{X}$-modules whose cohomology sheaves are coherent. By $\mathrm{D}_{\text {coh }}^{+}(X)$, $\mathrm{D}_{\text {coh }}^{-}(X)$, resp. $\mathrm{D}_{\text {coh }}^{\mathrm{b}}(X)$ we denote the full subcategories of $\mathrm{D}_{\text {coh }}(X)$ which consist of those objects whose cohomology vanishes in sufficiently negative degrees, resp. sufficiently positive degrees, resp. negative and positive degrees. Similarly, the notation $\mathrm{D}_{\mathrm{qc}}(X), \mathrm{D}_{\mathrm{qc}}^{\mathrm{b}}(X)$, $\mathrm{D}_{\mathrm{qc}}^{+}(X), \mathrm{D}_{\mathrm{qc}}^{-}(X)$ refers to quasi-coherent cohomology.

It is well-known that $\mathrm{D}_{\text {coh }}^{\mathrm{b}}(X)$ is equivalent to the derived category of the category of bounded complexes of coherent sheaves and similarly for $\mathrm{D}_{\text {coh }}^{-}(X)$, see e.g. [21].

\section{Relative Fourier-Mukai transforms}

The main result of this section is Theorem 2.12, which shows that a certain relative Fourier-Mukai transform is an equivalence of categories in two ways:

$$
\mathrm{FM}_{\mathcal{P}}^{-}: \mathrm{D}_{\text {coh }}^{-}(X) \rightarrow \mathrm{D}_{\text {coh }}^{-}(X) \text { and } \quad \mathrm{FM}_{\mathcal{P}}^{\mathrm{b}}: \mathrm{D}_{\text {coh }}^{\mathrm{b}}(X) \rightarrow \mathrm{D}_{\text {coh }}^{\mathrm{b}}(X)
$$

In Remark 2.13, we explain how the techniques developed in [27, 23] for unbounded complexes can be used to extend this result to obtain equivalences

$$
\mathrm{FM}_{\mathcal{P}}^{+}: \mathrm{D}_{\text {coh }}^{+}(X) \rightarrow \mathrm{D}_{\text {coh }}^{+}(X) \text { and } \mathrm{FM}_{\mathcal{P}}: \mathrm{D}_{\text {coh }}(X) \rightarrow \mathrm{D}_{\text {coh }}(X)
$$


Our main result will be shown under the following assumptions:

$S$ and $X$ are reduced, connected and separated schemes; $S$ is of finite type over $\boldsymbol{k}$;

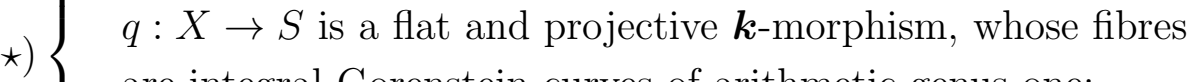
are integral Gorenstein curves of arithmetic genus one; there exists a section $\sigma: S \rightarrow X$ of $q$ which factors through the open set of points in $X$, at which $q$ is smooth.

If these assumptions are satisfied, the image of the section $\sigma$ is a Cartier divisor $\Sigma \subset X$. These assumptions imply that $S, X$ and $X \times_{S} X$ are Noetherian and of finite dimension. By $\Delta \subset X \times_{S} X$ we denote the diagonal and by $\mathcal{I}_{\Delta}$ its ideal sheaf. The diagonal embedding is denoted $\delta: X \rightarrow X \times{ }_{S} X$.

The sheaf $\mathcal{P}$ on $X \times_{S} X$, which is used in the definition of $\mathrm{FM}_{\mathcal{P}}$, is

$$
\mathcal{P}:=\mathcal{I}_{\Delta} \otimes \pi_{1}^{*} \mathcal{O}_{X}(\Sigma) \otimes \pi_{2}^{*} \mathcal{O}_{X}(\Sigma)
$$

The sheaf $\mathcal{P}$ is flat over both factors, because the diagonal has this property and $\mathcal{O}_{X}(\Sigma)$ is locally free.

We use the following notation. If $q: X \rightarrow S$ is a morphism of schemes and $s \in S, x \in X$ are points, the fibre of $q$ over $s$ is denoted by $X_{s}$ and its embedding into $X$ by $j_{s}: X_{s} \subset X$. We denote the two projections $X \times_{S} X \rightarrow X$ by $\pi_{1}, \pi_{2}$ and the two projections $X_{s} \times X_{s} \rightarrow X_{s}$ by $p_{1}, p_{2}$. Furthermore, we abbreviate $\pi:=q \circ \pi_{1}=q \circ \pi_{2}: X \times{ }_{S} X \rightarrow S$.

The fibres of both projections $\pi_{1}$ and $\pi_{2}$ over $x \in X$ are isomorphic to $X_{q(x)}$, so that we have two Cartesian squares:

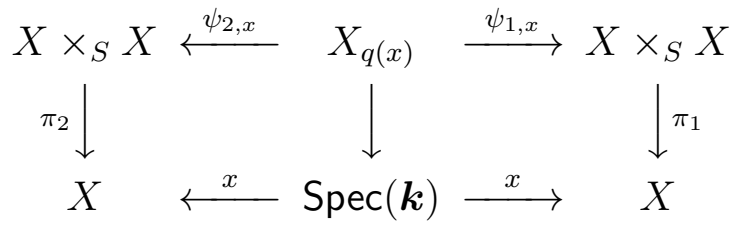

In addition, we have $\pi_{1} \circ \psi_{2, x}=\pi_{2} \circ \psi_{1, x}=j_{q(x)}$. The morphisms $\psi_{\nu, x}$ coincide with the compositions

$$
\psi_{\nu, x}: X_{q(x)} \rightarrow X_{q(x)} \times X_{q(x)} \rightarrow X \times{ }_{S} X,
$$

where the first map embeds $X_{q(x)}$ as the fibre of $p_{\nu}$ over $x$ and the second morphism is just $j_{q(x)} \times j_{q(x)}$. By $X_{s} \cong \Delta_{s} \subset X_{s} \times X_{s}$ we denote the diagonal and by $\mathcal{I}_{\Delta_{s}}$ its ideal sheaf.

Under the assumptions $(\star)$ we obtain for any $s \in S$ :

$$
\left(j_{s} \times j_{s}\right)^{*} \mathcal{P} \cong \mathcal{I}_{\Delta_{s}} \otimes p_{1}^{*} \mathcal{O}_{X_{s}}(\sigma(s)) \otimes p_{2}^{*} \mathcal{O}_{X_{s}}(\sigma(s))
$$


If $s=q(x)$, this implies

$$
\psi_{1, x}^{*} \mathcal{P} \cong \psi_{2, x}^{*} \mathcal{P} \cong \mathcal{I}_{x} \otimes \mathcal{O}_{X_{s}}(\sigma(s)),
$$

where $\mathcal{I}_{x}$ denotes the ideal sheaf of the point $x$ in $X_{s}=X_{q(x)}$. Because $\sigma(s)$ is smooth in its fibre $X_{s}$, as a special case we obtain

$$
\psi_{1, \sigma(s)}^{*} \mathcal{P} \cong \psi_{2, \sigma(s)}^{*} \mathcal{P} \cong \mathcal{O}_{X_{s}} .
$$

The following result is an immediate consequence of [1, Theorem 1.9].

Lemma 2.1. Let $T$ be a scheme, which is locally of finite type over $\boldsymbol{k}$, $f: Y \rightarrow T$ a projective and flat morphism of schemes and $\mathcal{P}$ a $T$-flat coherent sheaf on $Y$. Suppose $\mathcal{E} x t_{Y_{t}}^{i}\left(\mathcal{P}(t), \mathcal{O}_{Y_{t}}\right)=0$ for all points $t \in T$ and all $i>0$, where $Y_{t} \subset Y$ is the fibre of $f$ over $t$ and $\mathcal{P}(t)$ denotes the restriction of $\mathcal{P}$ to $Y_{t}$. Then, the following holds:

$$
\mathcal{E} x t_{Y}^{i}(\mathcal{P}, \mathcal{O})=0 \text { for } i>0 \text { and } \mathcal{P}^{\vee} \text { is } T \text {-flat. }
$$

Furthermore, for any morphism $g: T^{\prime} \rightarrow T$ there is an isomorphism

$$
\left(\mathbf{1}_{Y} \times g\right)^{*}\left(\mathcal{P}^{\vee}\right) \cong\left(\left(\mathbf{1}_{Y} \times g\right)^{*} \mathcal{P}\right)^{\vee}
$$

on $Y \times_{T} T^{\prime}$.

Corollary 2.2. Let $f: Y \rightarrow T$ be a projective and flat morphism of schemes, with $T$ locally of finite type over $\boldsymbol{k}$. Suppose, $f$ is a Gorenstein morphism of pure dimension one and $\mathcal{P}$ is a $T$-flat coherent sheaf on $Y$ such that for any point $t \in T$ the restriction $\mathcal{P}(t)$ of $\mathcal{P}$ to the fibre $Y_{t}$ is torsion free. Then, the following holds:

$$
\mathcal{E} x t_{Y}^{i}(\mathcal{P}, \mathcal{O})=0 \text { for } i>0 \text { and } \mathcal{P}^{\vee} \text { is } T \text {-flat. }
$$

Furthermore, for any morphism $g: T^{\prime} \rightarrow T$ there is an isomorphism

$$
\left(\mathbf{1}_{Y} \times g\right)^{*}\left(\mathcal{P}^{\vee}\right) \cong\left(\left(\mathbf{1}_{Y} \times g\right)^{*} \mathcal{P}\right)^{\vee}
$$

on $Y \times_{T} T^{\prime}$.

Proof. Because, by assumption, $\mathcal{P}(t)$ is torsion free on the curve $Y_{t}$, for any point $y \in Y_{t}$ we have $\operatorname{depth}\left(\mathcal{P}(t)_{y}\right) \geq 1$. Because $Y_{t}$ has pure dimension one, this implies that $\mathcal{P}(t)_{y}$ is a maximal Cohen-Macaulay module over the local Gorenstein ring $\mathcal{O}_{Y_{t}, y}$. Hence, by local duality [11. Cor. 3.5.11], we obtain $\mathcal{E} x t_{Y_{t}}^{i}\left(\mathcal{P}(t), \mathcal{O}_{Y_{t}}\right)=0$ for all $i>0$. The claim follows now from Lemma 2.1.

If the morphism $q: X \rightarrow S$ satisfies $(\star)$, we can apply the corollary to $f: Y \rightarrow T$ being either projection $\pi_{1}, \pi_{2}: X \times_{S} X \rightarrow X$.

In the course of the proof of the following proposition we use the compactified Picard scheme as it was introduced by A. Altman and S. Kleiman in [1] and 2]. Let us briefly recall some notation and the 
results we are going to use. Let $S$ be a scheme which is locally of finite type over $\boldsymbol{k}$ and let $q: X \rightarrow S$ be a flat and projective morphism, whose fibres $X_{s}$ are integral curves. Without the assumption on the fibres, there are two functors $\mathrm{Pic}_{X \mid S}^{-}$and $\mathrm{Pic}_{X \mid S}^{\overline{ }}$ defined, which associate to any $S$-scheme the set of all equivalence classes of flat families of torsion free rank one sheaves, which are, in addition, supposed to be Cohen-Macaulay on the fibres in the second case. Two such families are equivalent, if they differ by a twist with the pull-back of an invertible sheaf from the parameter scheme. Because on an integral curve a torsion free sheaf is automatically Cohen-Macaulay, these two functors coincide in our situation. We denote them by $\overline{\mathrm{Pic}}_{X \mid S}$.

A pair $(P, \mathcal{F})$, which consists of an $S$-scheme $P$ and a finitely presented sheaf $\mathcal{F}$ on $X \times{ }_{S} P$, which is $P$-flat and whose restrictions to fibres of the projection to $P$ are torsion free sheaves of rank one, is said to represent the functor $\overline{\mathrm{Pic}}_{X \mid S}$ if the following holds: for any $S$-scheme $T$ and any $T$-flat family $\mathcal{G}$ of finitely presented torsion free sheaves of rank one on $X \times{ }_{S} T$ there exists a unique $S$-morphism $f: T \rightarrow P$, such that there exists an invertible sheaf $\mathcal{A}$ on $T$ and an isomorphism $\left(\mathbf{1}_{X} \times f\right)^{*} \mathcal{F} \cong \mathcal{G} \otimes q_{T}^{*} \mathcal{A}$. By $q_{T}$ we denote here the second projection $X \times{ }_{S} T \rightarrow T$.

In general, the functor $\overline{\mathrm{Pic}}_{X \mid S}$ is not representable by an $S$-scheme, because it is not a sheaf in the Zariski topology or in any finer topology. Therefore, in general, this functor has to be sheafified. The sheafified functor in the étale topology was studied in [1]. However, under the assumption of the existence of a section $\sigma: S \rightarrow X$ of $q$, which factors through the smooth locus of $q$, it was shown in 2], Theorem 3.4 (iii), that the functor $\overline{\mathrm{Pic}}_{X \mid S}$ is a sheaf in the étale topology. Actually, they show it is a sheaf in the finer fppf-topology. Hence, we can apply [1], Theorems 8.1 and 8.5 , to conclude: $\overline{\mathrm{Pic}}_{X \mid S}$ is representable by an $S$-scheme, which is the disjoint union of infinitely many projective $S$-schemes $\overline{\mathrm{Pic}}_{X \mid S}^{n}$, which represent the functors $\overline{\mathrm{Pic}}_{X \mid S}^{n}$. The functor $\overline{\operatorname{Pic}}_{X \mid S}^{n}$ is the open sub-functor of $\overline{\operatorname{Pic}}_{X \mid S}$ which parametrises sheaves of degree $n$ on the fibres. All these $S$-schemes $\overline{\operatorname{Pic}}_{X \mid S}^{n}$ are isomorphic to each other, because the tensor product with the invertible sheaf $\pi_{1}^{*} \mathcal{O}_{X}(\Sigma)$ defines an isomorphism of functors $\overline{\mathrm{Pic}}_{X \mid S}^{n} \rightarrow \overline{\mathrm{Pic}}_{X \mid S}^{n+1}$.

Finally, assuming in addition that all fibres have arithmetic genus one, in [1], Example 8.9 (iii), it was shown that the functor $\overline{\mathrm{Pic}}_{X \mid S}^{-1}$ is represented by $\left(X, \mathcal{I}_{\Delta}\right)$. Hence, the functor $\overline{\mathrm{Pic}}_{X \mid S}^{0}$ is represented by any pair $\left(X, \mathcal{I}_{\Delta} \otimes \pi_{1}^{*} \mathcal{O}_{X}(\Sigma) \otimes \pi_{2}^{*} \mathcal{A}\right)$, where $\mathcal{A}$ is an invertible sheaf on 
$X$. In particular, if the assumptions $(\star)$ are satisfied, the pair $(X, \mathcal{P})$ represents the functor $\overline{\mathrm{Pic}}_{X \mid S}^{0}$.

If $g: T \rightarrow S$ is a morphism of schemes, we obtain an isomorphism of functors $\overline{\mathrm{Pic}}_{X \mid S}^{0} \times{ }_{S} T \cong \overline{\mathrm{Pic}}_{X \times_{S} T \mid T}^{0}$. Hence, $\left(X \times_{S} T,\left(\mathbf{1}_{X} \times g\right)^{*} \mathcal{P}\right)$ represents $\overline{\operatorname{Pic}}_{X \times \times_{S} T \mid T}^{0}$. Here we used the isomorphism $\left(X \times_{S} X\right) \times_{S} T \cong$ $\left(X \times_{S} T\right) \times_{T}\left(X \times_{S} T\right)$.

Proposition 2.3. Under the assumptions $(*)$ there exists a unique morphism $i: X \rightarrow X$ of $S$-schemes such that there exists an invertible sheaf $\mathcal{M}$ on $S$ and an isomorphism of sheaves on $X \times{ }_{S} X$

$$
\left(\mathbf{1}_{X} \times i\right)^{*} \mathcal{P} \cong \mathcal{P}^{\vee} \otimes \pi^{*} \mathcal{M}
$$

Furthermore, $i$ is compatible with base change, which means the following. If we denote for any morphism $g: T \rightarrow S$ by $i_{g}: X \times_{S} T \rightarrow$ $X \times_{S} T$ the map which is defined via universality by $\left(\left(\mathbf{1}_{X \times \times_{S} X} \times g\right)^{*} \mathcal{P}\right)^{\vee}$ on $\left(X \times_{S} X\right) \times_{S} T$, then $i_{g}=i \times \mathbf{1}_{T}$.

Proof. As seen above, the pair $(X, \mathcal{P})$ represents the functor $\overrightarrow{\mathrm{Pic}}_{X \mid S}^{0}$. Because $\mathcal{P}^{\vee}$ is $\pi_{2}$-flat and, by Corollary [2.2 $\psi_{2, x}^{*}\left(\mathcal{P}^{\vee}\right) \cong\left(\psi_{2, x}^{*}(\mathcal{P})\right)^{\vee}$ is torsion free and of degree zero, the universality of $(X, \mathcal{P})$ implies the existence of a unique morphism $i: X \rightarrow X$ of $S$-schemes which satisfies $\left(\mathbf{1}_{X} \times i\right)^{*} \mathcal{P} \cong \mathcal{P}^{\vee} \otimes \pi_{2}^{*} \mathcal{A}$ for some invertible sheaf $\mathcal{A}$ on $X$. Now, consider the Cartesian diagram, where $\sigma_{1}=(\sigma \circ q) \times \mathbf{1}_{X}$

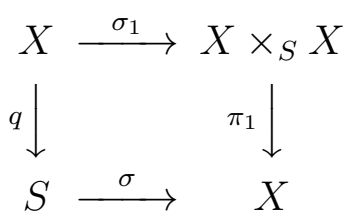

and restrict both sides of the isomorphism above to $X$ via $\sigma_{1}$. Because $\left(\mathbf{1}_{X} \times i\right) \circ \sigma_{1}=\sigma_{1} \circ i$ and $\pi_{2} \circ \sigma_{1}=\mathbf{1}_{X}$, this yields an isomorphism $i^{*} \sigma_{1}^{*} \mathcal{P} \cong \sigma_{1}^{*} \mathcal{P}^{\vee} \otimes \mathcal{A}$. Note that Corollary 2.2 yields an isomorphism $\sigma_{1}^{*}\left(\mathcal{P}^{\vee}\right) \cong\left(\sigma_{1}^{*} \mathcal{P}\right)^{\vee}$. But $\sigma_{1} \circ j_{s}=\psi_{1, \sigma(s)}$, hence $j_{s}^{*} \sigma_{1}^{*} \mathcal{P} \cong \psi_{1, \sigma(s)}^{*} \mathcal{P} \cong \mathcal{O}_{X_{s}}$ by (4), and $\mathcal{A}$ must be trivial on the fibres of $q$. This implies that there exists an invertible sheaf $\mathcal{M}$ on $S$, such that $\mathcal{A} \cong q^{*} \mathcal{M}$.

If $g: T \rightarrow S$ is an arbitrary morphism of schemes, from Corollary 2.2 we obtain $\left(\mathbf{1}_{X \times_{S} X} \times g\right)^{*}\left(\mathcal{P}^{\vee}\right) \cong\left(\left(\mathbf{1}_{X \times_{S} X} \times g\right)^{*} \mathcal{P}\right)^{\vee}$. The definition of $i, \overline{\mathrm{Pic}}_{X \times_{S} T \mid T}^{0} \cong \overline{\mathrm{Pic}}_{X \mid S} \times_{S} T$ and the uniqueness of $i_{g}$ imply now the compatibility with base change.

Remark 2.4. Suppose, the morphism $g$ in the proposition is given by a point $s \in S$, considered as a morphism $s: \operatorname{Spec}(\boldsymbol{k}) \rightarrow S$. Then, the induced morphism $i_{s}: X_{s} \rightarrow X_{s}$ coincides with the morphism $i$ used in [12, if the reference point $p_{0}$ is chosen to be equal to $\sigma(s)$. 
Lemma 2.5. Assuming $(\star)$, we obtain $\mathcal{P}^{\vee \vee} \cong \mathcal{P}, i^{2}=\mathbf{1}_{X}$ and $i$ is an isomorphism.

Proof. Here, we denote by $\mathcal{P}(x)$ the restriction of $\mathcal{P}$ to the fibre $X_{q(x)}$ of $\pi_{2}: X \times_{S} X \rightarrow X$ over $x \in X$. This is a torsion free sheaf on a Gorenstein curve, hence local duality applies to prove reflexivity of $\mathcal{P}(x)$. A local calculation shows that the canonical mappings

$$
\mathcal{P}(x) \rightarrow \mathcal{P}^{\vee \vee}(x) \rightarrow \mathcal{P}^{\vee}(x)^{\vee}
$$

and

$$
\mathcal{P}(x) \rightarrow \mathcal{P}(x)^{\vee \vee} \rightarrow \mathcal{P}^{\vee}(x)^{\vee}
$$

coincide. From Corollary 2.2 we know the second arrow in both cases is an isomorphism. Now, we obtain that the canonical isomorphism $\mathcal{P}(x) \rightarrow \mathcal{P}(x)^{\vee \vee}$ is isomorphic to the restriction of the canonical morphism of sheaves $\mathcal{P} \rightarrow \mathcal{P}^{\vee \vee}$. By Nakayama's lemma this morphism is surjective, whence we have an exact sequence

$$
0 \rightarrow \mathcal{K} \rightarrow \mathcal{P} \rightarrow \mathcal{P}^{\vee \vee} \rightarrow 0
$$

But, by Corollary 2.2, $\mathcal{P}^{\vee \vee}$ is $\pi_{2}$-flat, hence the restriction of (15) to the fibre $X_{q(x)}$ is exact. Reflexivity of $\mathcal{P}(x)$ implies $\mathcal{K}(x)=0$, and using Nakayama's lemma again, we obtain $\mathcal{K}=0$. Hence $\mathcal{P}$ is reflexive. The statement about $i^{2}$ is now an easy consequence of universality.

Remark 2.6. It is interesting to observe that the sheaf $\mathcal{P}$ is CohenMacaulay, provided the assumptions $(\star)$ are satisfied and $S$ itself is Cohen-Macaulay. This follows from [11, Theorem 2.1.7, because $\mathcal{P}$ is Cohen-Macaulay on all fibres as seen in the proof of Corollary 2.2. By the same reason, $X$ is Cohen-Macaulay if $S$ has this property.

Let $q: X \rightarrow S$ be a morphism which satisfies the conditions $(\star)$. We are going to study several versions of integral transforms $\mathrm{FM}_{\mathcal{Q}} \bullet$, defined by the formula

$$
\mathrm{FM}_{\mathcal{Q}} \cdot\left(\mathcal{E}^{\bullet}\right)=\boldsymbol{R} \pi_{2 *}\left(\mathcal{Q}^{\bullet} \stackrel{\boldsymbol{L}}{\otimes} \pi_{1}^{*}\left(\mathcal{E}^{\bullet}\right)\right) .
$$

If an integral transform $\mathrm{FM}_{\mathcal{Q}}$ • is an equivalence of categories, it is called a Fourier-Mukai transform. The object $\mathcal{Q}^{\bullet}$ is called the kernel of the integral transform $\mathrm{FM}_{\mathcal{Q}} \bullet$

Remark 2.7. Because $X \times_{S} X$ is Noetherian and of finite dimension, the functor $\pi_{2 *}$ has finite cohomological dimension. Hence, $\boldsymbol{R} \pi_{2 *}$ is way-out in both directions and [20, I $\S 7$, allows to define $\boldsymbol{R} \pi_{2 *}$ for unbounded complexes. Moreover, $\pi_{2 *}$ is proper, hence we obtain

$$
\boldsymbol{R} \pi_{2 *}: \mathrm{D}_{\text {coh }}\left(X \times_{S} X\right) \rightarrow \mathrm{D}_{\text {coh }}(X)
$$


with restrictions $\mathrm{D}_{\text {coh }}^{+}\left(X \times_{S} X\right) \rightarrow \mathrm{D}_{\text {coh }}^{+}(X), \mathrm{D}_{\text {coh }}^{-}\left(X \times_{S} X\right) \rightarrow \mathrm{D}_{\text {coh }}^{-}(X)$ and $\mathrm{D}_{\text {coh }}^{\mathrm{b}}\left(X \times_{S} X\right) \rightarrow \mathrm{D}_{\text {coh }}^{\mathrm{b}}(X)$. Similar remarks apply to the other projections which we use below. Moreover, from [20, II.5.1, we obtain an isomorphism

$$
\boldsymbol{R}\left(\pi_{2 *} \circ \pi_{23 *}\right) \cong \boldsymbol{R} \pi_{2 *} \circ \boldsymbol{R} \pi_{23 *}
$$

of functors $\mathrm{D}_{\text {coh }}\left(X \times_{S} X \times_{S} X\right) \rightarrow \mathrm{D}_{\text {coh }}(X)$.

To deal with unbounded complexes when deriving the tensor product is more difficult, because we normally don't have a finiteness condition as above. In [20], II $\S 4$, we find a definition of the derived tensor product

$$
\stackrel{L}{\otimes}: \mathrm{D}_{\text {coh }}^{-}(X) \times \mathrm{D}_{\text {coh }}^{-}(X) \rightarrow \mathrm{D}_{\text {coh }}^{-}(X) .
$$

If $f: X \rightarrow Y$ is a morphism and $Y$ is locally Noetherian, there exists a derived functor $\boldsymbol{L} f^{*}: \mathrm{D}_{\text {coh }}^{-}(Y) \rightarrow \mathrm{D}_{\text {coh }}^{-}(X)$, [20, II $\S 4$. It satisfies $\boldsymbol{L}\left(f^{*} g^{*}\right) \cong \boldsymbol{L} f^{*} \boldsymbol{L} g^{*}$. Moreover, if $f$ has finite Tor-dimension, e.g. if $f$ is flat, these are available for unbounded complexes with coherent cohomology. If $f: X \rightarrow Y$ is arbitrary and $\mathcal{F}^{\bullet}, \mathcal{G}^{\bullet} \in \mathrm{D}^{-}(Y)$, there is an isomorphism $\boldsymbol{L} f^{*} \mathcal{F}^{\bullet} \stackrel{\boldsymbol{L}}{\otimes} \boldsymbol{L} f^{*} \mathcal{G}^{\bullet} \cong \boldsymbol{L} f^{*}\left(\mathcal{F}^{\bullet} \stackrel{\boldsymbol{L}}{\otimes} \mathcal{G}^{\bullet}\right)$, see [20], II $\S 5$.

Finally, the projection formula

$$
\boldsymbol{R} f_{*} \mathcal{F}^{\bullet} \stackrel{\boldsymbol{L}}{\otimes} \mathcal{G}^{\bullet} \cong \boldsymbol{R} f_{*}\left(\mathcal{F}^{\bullet} \stackrel{\boldsymbol{L}}{\otimes} \boldsymbol{L} f^{*} \mathcal{G}^{\bullet}\right)
$$

is a very important tool in our calculations. In [20, II $\S 5$, this isomorphism is proved with $\mathcal{F}^{\bullet} \in \mathrm{D}_{\text {coh }}^{-}(X), \mathcal{G}^{\bullet} \in \mathrm{D}_{\text {coh }}^{-}(Y)$ and $f: X \rightarrow Y$ a quasi-compact morphism of Noetherian schemes of finite dimension.

With this preparation in mind, for any $\mathcal{Q}^{\bullet} \in \mathrm{D}_{\text {coh }}^{-}\left(X \times_{S} X\right)$ it is now clear that the formula (6) defines a functor

$$
\mathrm{FM}_{\mathcal{Q}}^{-} \bullet \mathrm{D}_{\text {coh }}^{-}(X) \rightarrow \mathrm{D}_{\text {coh }}^{-}(X)
$$

If $\mathcal{Q}^{\bullet}$ is isomorphic to a bounded complex of coherent $\pi_{1}$-flat sheaves with $\pi_{2}$-proper support, it was shown in [14 that the restriction of $\mathrm{FM}_{\mathcal{Q}}^{-} \cdot$ is a functor

$$
\mathrm{FM}_{\mathcal{Q}}^{\mathrm{b}} \cdot \mathrm{D}_{\mathrm{coh}}^{\mathrm{b}}(X) \rightarrow \mathrm{D}_{\mathrm{coh}}^{\mathrm{b}}(X) .
$$

See Remark 2.13 for a discussion how to extend the results of [20] and the definition of $\mathrm{FM}_{\mathcal{Q}}$ to unbounded complexes.

Example 2.8. Let $\mathcal{L}$ be a line bundle on $X$ and recall that $\delta: X \rightarrow$ $X \times_{S} X$ denotes the diagonal embedding. In this case, $\delta_{*} \mathcal{L}$ is $\pi_{1}$-flat. Because $\boldsymbol{R} \pi_{2 *}\left(\delta_{*} \mathcal{L} \stackrel{\boldsymbol{L}}{\otimes} \pi_{1}^{*} \mathcal{E}^{\bullet}\right) \cong \boldsymbol{R} \pi_{2 *}\left(\delta_{*}\left(\mathcal{L} \otimes \boldsymbol{L} \delta^{*} \pi_{1}^{*} \mathcal{E}^{\bullet}\right)\right) \cong \mathcal{L} \otimes \mathcal{E}^{\bullet}$, we obtain

$$
\mathrm{FM}_{\delta_{*} \mathcal{L}}^{-}\left(\mathcal{E}^{\bullet}\right) \cong \mathcal{L} \otimes \mathcal{E}^{\bullet} \quad \text { and } \quad \mathrm{FM}_{\delta_{*} \mathcal{L}}^{\mathrm{b}}\left(\mathcal{E}^{\bullet}\right) \cong \mathcal{L} \otimes \mathcal{E}^{\bullet}
$$


Here, we used $\pi_{2} \circ \delta=\mathbf{1}_{X}=\pi_{1} \circ \delta$ and the projection formula

$$
\delta_{*} \mathcal{L} \stackrel{L}{\otimes} \mathcal{G}^{\bullet} \cong \delta_{*}\left(\mathcal{L} \otimes \boldsymbol{L} \delta^{*} \mathcal{G}^{\bullet}\right)
$$

with $\mathcal{G}^{\bullet}=\pi_{1}^{*} \mathcal{E}^{\bullet}$. Note that $\delta_{*}$ and $\mathcal{L} \otimes(\cdot)$ are exact functors. In [20], II.5.6, this formula is shown for $\mathcal{G}^{\bullet} \in \mathrm{D}_{\text {coh }}^{-}\left(X \times_{S} X\right)$. Therefore, we don't get from [20] the corresponding result for $\mathrm{FM}_{\delta_{*} \mathcal{L}}^{+}$or $\mathrm{FM}_{\delta_{*} \mathcal{L}}$. See, however, Remark 2.13.

Observe that $\mathrm{FM}_{\mathcal{Q}}^{-}$. commutes with shift functors, that is

$$
\mathrm{FM}_{\mathcal{Q}}^{-} \bullet[m] \cong[m] \circ \mathrm{FM}_{\mathcal{Q}}^{-} \cong \mathrm{FM}_{\mathcal{Q} \bullet[m]}^{-}
$$

for all $m \in \mathbb{Z}$. Moreover, the flat base change theorem implies for any flat $S$-morphism $f: X \rightarrow X$ that there exists an isomorphism of functors

$$
f^{*} \circ \mathrm{FM}_{\mathcal{Q}}^{-} \cong \mathrm{FM}_{\left(1_{X} \times f\right)^{*} \mathcal{Q}}^{-} \cdot
$$

In the following lemmas we collected less obvious properties of integral transforms, which are needed in the proof of our main result below.

Lemma 2.9. Suppose $S$ is of finite type over $\boldsymbol{k}$, let $q: X \rightarrow S$ be a flat and proper morphism of schemes and $\eta: Y \rightarrow X$ a closed subscheme. Suppose $\zeta: Z \rightarrow X$ is a closed subscheme such that the diagram

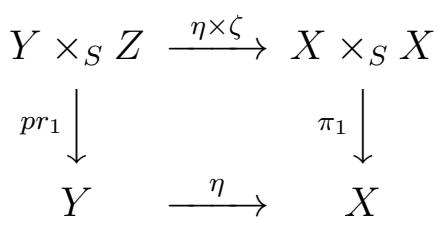

is Cartesian, e.g. $Z=q^{-1}(q(Y))$. Suppose $\mathcal{Q}^{\bullet} \in \mathrm{D}_{\text {coh }}^{-}\left(X \times_{S} X\right)$ and denote its derived restriction by $\mathcal{R}^{\bullet}=\boldsymbol{L}(\eta \times \zeta)^{*} \mathcal{Q}^{\bullet}$. Then, there is an isomorphism of functors $\mathrm{D}_{\text {coh }}^{-}(Y) \rightarrow \mathrm{D}_{\text {coh }}^{-}(X)$

$$
\mathrm{FM}_{\mathcal{Q}}^{-} \circ \eta_{*} \cong \zeta_{*} \circ \mathrm{FM}_{\mathcal{R}}^{-} \cdot
$$

Proof. In the commutative diagram

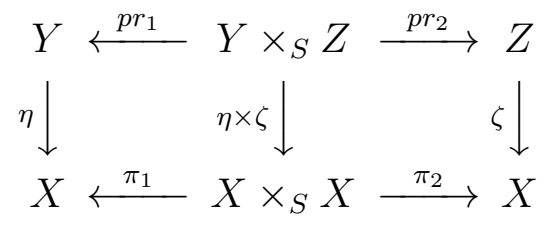

the left square is Cartesian by assumption. Because $\pi_{1}$ and, hence, $p r_{1}$ are flat, from this Cartesian diagram we obtain an isomorphism of functors

$$
\pi_{1}^{*} \circ \eta_{*} \cong(\eta \times \zeta)_{*} \circ p r_{1}^{*}
$$


The four functors involved in the isomorphism (8) are exact, hence they coincide with their derived versions. Using that closed embeddings are proper, we obtain that (8) is an isomorphism of functors $\mathrm{D}_{\text {coh }}^{-}(Y) \rightarrow$ $\mathrm{D}_{\text {coh }}^{-}\left(X \times_{S} X\right)$.

Because the schemes considered here are of finite dimension, and because $\eta \times \zeta$ is affine, which implies exactness of $(\eta \times \zeta)_{*}$, the projection formula gives a functorial isomorphism

$$
\mathcal{Q}^{\bullet} \stackrel{\boldsymbol{L}}{\otimes}(\eta \times \zeta)_{*} \mathcal{F}^{\bullet} \cong(\eta \times \zeta)_{*}\left(\boldsymbol{L}(\eta \times \zeta)^{*} \mathcal{Q}^{\bullet} \stackrel{\boldsymbol{L}}{\otimes} \mathcal{F}^{\bullet}\right)
$$

with $\mathcal{F}^{\bullet} \in \mathrm{D}_{\text {coh }}^{-}\left(Y \times_{S} Z\right)$ and $\mathcal{Q}^{\bullet} \in \mathrm{D}_{\text {coh }}^{-}\left(X \times_{S} X\right)$.

Finally, from commutativity of the right hand square in the diagram (17) together with exactness of the functors $\zeta_{*}$ and $(\eta \times \zeta)_{*}$ we gain an isomorphism of functors $\mathrm{D}_{\text {coh }}^{-}\left(Y \times{ }_{S} Z\right) \rightarrow \mathrm{D}_{\text {coh }}^{-}(X)$

$$
\boldsymbol{R} \pi_{2 *} \circ(\eta \times \zeta)_{*} \cong \zeta_{*} \circ \boldsymbol{R} p r_{2 *} .
$$

Putting these observations together, we obtain the desired isomorphism, which is functorial in $\mathcal{E}^{\bullet} \in \mathrm{D}_{\text {coh }}^{-}(Y)$.

$$
\begin{array}{rlr}
\mathrm{FM}_{\mathcal{Q}}^{-}\left(\eta_{*} \mathcal{E}^{\bullet}\right) & \cong \boldsymbol{R} \pi_{2 *}\left(\mathcal{Q}^{\bullet} \stackrel{\boldsymbol{L}}{\otimes} \pi_{1}^{*} \eta_{*} \mathcal{E}^{\bullet}\right) & \\
& \cong \boldsymbol{R} \pi_{2 *}\left(\mathcal{Q}^{\bullet} \stackrel{\boldsymbol{L}}{\otimes}(\eta \times \zeta)_{*} p r_{1}^{*} \mathcal{E}^{\bullet}\right) & \text { using (8) } \\
& \cong \boldsymbol{R} \pi_{2 *}\left((\eta \times \zeta)_{*}\left(\boldsymbol{L}(\eta \times \zeta)^{*} \mathcal{Q}^{\bullet} \stackrel{\boldsymbol{L}}{\otimes} p r_{1}^{*} \mathcal{E}^{\bullet}\right)\right) & \text { using (9]) } \\
& \cong \zeta_{*} \boldsymbol{R} p r_{2 *}\left(\mathcal{R}^{\bullet} \stackrel{\boldsymbol{L}}{\otimes} p r_{1}^{*} \mathcal{E}^{\bullet}\right) & \text { using (10) } \\
& \cong \zeta_{*}\left(\mathrm{FM}_{\mathcal{R}}^{-}\left(\mathcal{E}^{\bullet}\right)\right) .
\end{array}
$$

As a corollary, we obtain a useful and probably well-known result, a version of which can be found in [14, Lemma 6.1.

Corollary 2.10. Suppose $S$ is of finite type over $\boldsymbol{k}, s \in S$ and $q: X \rightarrow$ $S$ is flat and projective. Let $\mathcal{Q}^{\bullet} \in \mathrm{D}_{\text {coh }}^{-}\left(X \times_{S} X\right)$ and denote by $\mathcal{Q}_{s}^{\bullet}:=$ $\boldsymbol{L}\left(j_{s} \times j_{s}\right)^{*} \mathcal{Q}^{\bullet}$ its derived restriction. Then, there is an isomorphism of functors $\mathrm{D}_{\text {coh }}^{-}\left(X_{s}\right) \rightarrow \mathrm{D}_{\text {coh }}^{-}(X)$

$$
j_{s *} \circ \mathrm{FM}_{\mathcal{Q}_{s}}^{-} \cong \mathrm{FM}_{\mathcal{Q}}^{-} \circ j_{s *} .
$$

Proof. This follows with $Y=Z=X_{s}$ and $\zeta=\eta=j_{s}$ from Lemma 2.9 .

Lemma 2.11. Let $S$ be of finite type over $\boldsymbol{k}, X$ connected and reduced and $q: X \rightarrow S$ a flat, projective morphism. If $\mathcal{Q}^{\bullet} \in \mathrm{D}_{\text {coh }}^{\mathrm{b}}\left(X \times_{S} X\right)$ is such that $\mathrm{FM}_{\mathcal{Q}}^{-} \cdot(\boldsymbol{k}(x)) \cong \boldsymbol{k}(x)$ for all points $x \in X$, there exists a line 
bundle $\mathcal{L}$ on $X$ such that $\mathcal{Q}^{\bullet} \cong \delta_{*} \mathcal{L}$. This implies $\operatorname{FM}_{\mathcal{Q}}^{-} \cdot\left(\mathcal{E}^{\bullet}\right) \cong \mathcal{E}^{\bullet} \otimes \mathcal{L}$, functorial in $\mathcal{E}^{\bullet} \in \mathrm{D}_{\text {coh }}^{-}(X)$.

Proof. By assumption, we know $\mathrm{FM}_{\mathcal{Q}}^{-} \cdot(\boldsymbol{k}(x))=\boldsymbol{R} \pi_{2 *}\left(\mathcal{Q}^{\bullet} \stackrel{\boldsymbol{L}}{\otimes} \pi_{1}^{*} \boldsymbol{k}(x)\right)$ $\cong \boldsymbol{R} \pi_{2 *} \psi_{1, x *} \boldsymbol{L} \psi_{1, x}^{*} \mathcal{Q}^{\bullet} \cong j_{q(x) *} \boldsymbol{L} \psi_{1, x}^{*} \mathcal{Q}^{\bullet}$ is isomorphic to $\boldsymbol{k}(x)$. Hence, $\boldsymbol{L} \psi_{1, x}^{*} \mathcal{Q}^{\bullet}$ is a sheaf for all points $x \in X$. From [8], Lemma 4.3 we deduce now that $\mathcal{Q}^{\bullet}$ is a coherent sheaf $\mathcal{Q}$ on $X \times_{S} X$ which is $\pi_{1}$-flat. In particular, $\boldsymbol{L} \psi_{1, x}^{*} \mathcal{Q} \cong \psi_{1, x}^{*} \mathcal{Q} \cong \boldsymbol{k}(x)$.

Because $q$ is projective, there is a $q$-very ample line bundle $\mathcal{A}$ on $X$ and $\pi_{2}^{*} \mathcal{A}$ is $\pi_{1}$-ample. For large positive $m$ the canonical mapping

$$
\pi_{1}^{*} \pi_{1 *}\left(\mathcal{Q} \otimes \pi_{2}^{*} \mathcal{A}^{\otimes m}\right) \rightarrow \mathcal{Q} \otimes \pi_{2}^{*} \mathcal{A}^{\otimes m}
$$

is surjective and $R^{j} \pi_{1 *}\left(\mathcal{Q} \otimes \pi_{2}^{*} \mathcal{A}^{\otimes m}\right)=0$ for any $j>0$.

From $H^{j}\left(\psi_{1, x}^{*}\left(\mathcal{Q} \otimes \pi_{2}^{*} \mathcal{A}^{\otimes m}\right)\right) \cong H^{j}\left(\psi_{1, x}^{*} \mathcal{Q} \otimes j_{q(x)}^{*} \mathcal{A}^{\otimes m}\right) \cong H^{j}(\boldsymbol{k}(x) \otimes$ $\left.j_{q(x)}^{*} \mathcal{A}^{\otimes m}\right) \cong H^{j}(\boldsymbol{k}(x))$, we obtain

$$
h^{j}\left(\psi_{1, x}^{*}\left(\mathcal{Q} \otimes \pi_{2}^{*} \mathcal{A}^{\otimes m}\right)\right)= \begin{cases}1 & \text { if } j=0, \\ 0 & \text { if } j \neq 0 .\end{cases}
$$

Hence, $\mathcal{B}:=\pi_{1 *}\left(\mathcal{Q} \otimes \pi_{2}^{*} \mathcal{A}^{\otimes m}\right)$ is locally free of rank one on $X$. Therefore, from (11) we obtain a surjection

$$
\mathcal{O}_{X \times{ }_{S} X} \rightarrow \mathcal{Q} \otimes \pi_{1}^{*} \mathcal{B}^{\vee} \otimes \pi_{2}^{*} \mathcal{A}^{\otimes m}
$$

Again, $\psi_{1, x}^{*}\left(\mathcal{Q} \otimes \pi_{1}^{*} \mathcal{B}^{\vee} \otimes \pi_{2}^{*} \mathcal{A}^{\otimes m}\right) \cong \boldsymbol{k}(x)$, which implies the existence of a unique $S$-morphism $\varphi: X \rightarrow \operatorname{Hilb}^{1}(X \mid S)$ such that (12) is isomorphic to the pull-back $\left(\mathbf{1}_{X} \times \varphi\right)^{*}\left(\mathcal{O}_{X \times \operatorname{Hilb}^{1}(X \mid S)} \rightarrow \mathcal{U}\right)$ of the universal quotient. But, by [1], Lemma 8.7, $\operatorname{Hilb}^{1}(X \mid S)$ is represented by $\left(X, \mathcal{O}_{\Delta}\right)$, hence $\varphi: X \rightarrow X$ satisfies $\left(\varphi \times \mathbf{1}_{X}\right)^{*} \mathcal{O}_{\Delta} \cong \mathcal{Q} \otimes \pi_{1}^{*} \mathcal{B}^{\vee} \otimes \pi_{2}^{*} \mathcal{A}^{\otimes m}$. Because (12) restricted to the fibre of $\pi_{1}$ over $x$ is the canonical map $\mathcal{O}_{X_{q(x)}} \rightarrow \boldsymbol{k}(x)$, we must have $\varphi=\mathbf{1}_{X}$. Hence, $\mathcal{Q} \cong \mathcal{O}_{\Delta} \otimes \pi_{2}^{*}\left(\mathcal{B} \otimes \mathcal{A}^{\otimes-m}\right)$. With $\mathcal{L}:=\mathcal{B} \otimes \mathcal{A}^{\otimes-m}$ the claim now follows.

Our main focus is on the integral transform whose kernel is the $\pi_{1}$ flat sheaf $\mathcal{P}$ on $X \times_{S} X$, which was defined in (1). In this case, we actually obtain a functor

$$
\mathrm{FM}_{\mathcal{P}}: \mathrm{D}_{\mathrm{coh}}(X) \rightarrow \mathrm{D}_{\mathrm{coh}}(X)
$$

This follows because $\mathcal{P} \otimes \pi_{1}^{*}(\cdot)$ is an exact functor and, as seen before, $\boldsymbol{R} \pi_{2 *}$ is defined for unbounded complexes. Moreover, the restriction to 
the full sub-categories $\mathrm{D}_{\text {coh }}^{\mathrm{b}}(X), \mathrm{D}_{\text {coh }}^{+}(X)$ and $\mathrm{D}_{\text {coh }}^{-}(X)$ define functors

$$
\begin{aligned}
& \mathrm{FM}_{\mathcal{P}}^{\mathrm{b}}: \mathrm{D}_{\text {coh }}^{\mathrm{b}}(X) \rightarrow \mathrm{D}_{\text {coh }}^{\mathrm{b}}(X) \\
& \mathrm{FM}_{\mathcal{P}}^{+}: \mathrm{D}_{\text {coh }}^{+}(X) \rightarrow \mathrm{D}_{\text {coh }}^{+}(X) \\
& \mathrm{FM}_{\mathcal{P}}^{-}: \mathrm{D}_{\text {coh }}^{-}(X) \rightarrow \mathrm{D}_{\text {coh }}^{-}(X) .
\end{aligned}
$$

In the definition of all of them, the derived tensor product is the ordinary one, because $\mathcal{P}$ is $\pi_{1}$-flat.

Theorem 2.12. If the assumptions ( $\star$ ) hold,

$$
\mathrm{FM}_{\mathcal{P}}^{-}: \mathrm{D}_{\text {coh }}^{-}(X) \rightarrow \mathrm{D}_{\text {coh }}^{-}(X) \quad \text { and } \quad \mathrm{FM}_{\mathcal{P}}^{\mathrm{b}}: \mathrm{D}_{\text {coh }}^{\mathrm{b}}(X) \rightarrow \mathrm{D}_{\text {coh }}^{\mathrm{b}}(X)
$$

are equivalences.

Proof. A standard calculation, see [25, 14, 6], shows

$$
\mathrm{FM}_{\mathcal{P}}^{-} \circ \mathrm{FM}_{\mathcal{P}}^{-} \cong \mathrm{FM}_{\mathcal{Q}^{\prime \bullet}}^{-} \quad \text { and } \quad \mathrm{FM}_{\mathcal{P}}^{\mathrm{b}} \circ \mathrm{FM}_{\mathcal{P}}^{\mathrm{b}} \cong \mathrm{FM}_{\mathcal{Q}^{\prime \bullet}}^{\mathrm{b}},
$$

where $\pi_{i j}: X \times{ }_{S} X \times{ }_{S} X \rightarrow X \times{ }_{S} X$ denote the projections and $\mathcal{Q}^{\prime \bullet} \cong$ $\boldsymbol{R} \pi_{13 *}\left(\pi_{12}^{*} \mathcal{P} \otimes \pi_{23}^{*} \mathcal{P}\right)$ in both cases. All the necessary relations between derived functors, which are needed to calculate $\mathcal{Q}^{\prime}$, are contained in 20], II $\S 5$, provided we deal with complexes which are bounded above. The main ingredient, which is not provided by [20] for complexes which are bounded below or unbounded is the projection formula, see Remark 2.13 .

Observe that $\mathcal{Q}^{\prime \bullet}$ is an object of $\mathrm{D}_{\text {coh }}^{\mathrm{b}}\left(X \times_{S} X\right)$ and that the tensor product is the usual one, because $\mathcal{P}$ is $\pi_{1}$-flat. With

$$
\mathcal{Q}^{\bullet}:=\left(\mathbf{1}_{X} \times i\right)^{*} \mathcal{Q}^{\bullet}[1] \in \mathrm{D}_{\mathrm{coh}}^{\mathrm{b}}\left(X \times_{S} X\right)
$$

we obtain

$$
[1] \circ i^{*} \circ \mathrm{FM}_{\mathcal{P}}^{-} \circ \mathrm{FM}_{\mathcal{P}}^{-} \cong \mathrm{FM}_{\mathcal{Q}}^{-}
$$

and the same for $\mathrm{FM}^{\mathrm{b}}$. Our strategy is now to show $\mathrm{FM}_{\mathcal{Q}}^{-} \cdot(\boldsymbol{k}(x)) \cong$ $\boldsymbol{k}(x)$ for all $x \in X$. This enables us to calculate $\mathcal{Q}^{\bullet}$ with the aid of Lemma 2.11, hence gives us $\mathrm{FM}_{\mathcal{Q}}^{\mathrm{b}}$ as well. By Corollary 2.10, we have $\mathrm{FM}_{\mathcal{Q}}^{-} \circ j_{s *} \cong[1] \circ i^{*} \circ \mathrm{FM}_{\mathcal{P}}^{-} \circ \mathrm{FM}_{\mathcal{P}}^{-} \circ j_{s *} \cong[1] \circ j_{s *} \circ i_{s}^{*} \circ \mathrm{FM}_{\mathcal{P}_{s}}^{-} \circ \mathrm{FM}_{\mathcal{P}_{s}}^{-}$for all $s \in S$. Now, because $\mathcal{P}$ is $\pi_{1}$-flat and $q$ is flat by assumption, the sheaf $\mathcal{P}$ is $\pi$-flat as well. Hence, the derived restriction $\mathcal{P}_{s}$ to the fibre of $\pi$ over $s \in S$ coincides with the usual restriction $\left(j_{s} \times j_{s}\right)^{*} \mathcal{P}$. By (2), Remark 2.4 and [12], Remark 2.17, we see that [12] applied to the curve $X_{s}$ studies the functor $\mathbb{F}:=[1] \circ \mathrm{FM}_{\mathcal{P}_{s}}^{\mathrm{b}}$. This implies that [12], Theorem 2.18 gives an isomorphism $i_{s}^{*} \circ \mathrm{FM}_{\mathcal{P}_{s}}^{\mathrm{b}} \circ \mathrm{FM}_{\mathcal{P}_{s}}^{\mathrm{b}} \circ[1] \cong \mathbf{1}$. Therefore, we obtain

$$
\mathrm{FM}_{\mathcal{Q}}^{\mathrm{b}} \circ j_{s *} \cong j_{s *}
$$


Hence, $\mathrm{FM}_{\mathcal{Q}}^{-} \cdot(\boldsymbol{k}(x)) \cong \mathrm{FM}_{\mathcal{Q}}^{\mathrm{b}} \cdot(\boldsymbol{k}(x)) \cong \boldsymbol{k}(x)$ for all $x \in X$, and Lemma 2.11 implies the existence of a line bundle $\mathcal{L}$ on $X$ such that $\mathcal{Q}^{\bullet} \cong \delta_{*} \mathcal{L}$. Example 2.8 implies now $\mathrm{FM}_{\mathcal{Q}}^{-}\left(\mathcal{E}^{\bullet}\right) \cong \mathcal{E}^{\bullet} \otimes \mathcal{L}$ and $\mathrm{FM}_{\mathcal{Q}^{\bullet}}^{\mathrm{b}}\left(\mathcal{E}^{\bullet}\right) \cong \mathcal{E} \bullet \otimes \mathcal{L}$. With $\mathcal{E}^{\bullet}=j_{s *} \mathcal{O}_{X_{s}}$ and using (13), we see that $\mathcal{L}$ is trivial along the fibres of $q: X \rightarrow S$, hence $\mathcal{L} \cong q^{*} \mathcal{N}$ with some line bundle $\mathcal{N}$ on $S$. The functor $\mathbb{T}_{\mathcal{N}}$, which is the tensor product with $q^{*} \mathcal{N}$, is an equivalence on $\mathrm{D}_{\text {coh }}^{\mathrm{b}}(X)$ and on $\mathrm{D}_{\text {coh }}^{-}(X)$ and it commutes with $i^{*}$ and the shift functor $[-1]$. Therefore,

$$
\mathrm{FM}_{\mathcal{P}}^{-} \circ \mathrm{FM}_{\mathcal{P}}^{-} \cong \mathbb{T}_{\mathcal{N}} \circ i^{*} \circ[-1]
$$

which is an equivalence. The same statement is true for $F M^{b}$. This implies $\mathrm{FM}_{\mathcal{P}}^{-}$and $\mathrm{FM}_{\mathcal{P}}^{\mathrm{b}}$ are equivalences as well.

Remark 2.13. The results, which are collected in Remark 2.7 have been sufficient to prove that $\mathrm{FM}_{\mathcal{P}}^{-}$and $\mathrm{FM}_{\mathcal{P}}^{\mathrm{b}}$ are equivalences. However, if we like to extend this result to $\mathrm{FM}_{\mathcal{P}}^{+}$or $\mathrm{FM}_{\mathcal{P}}$, we need a definition of the derived tensor product, a formula for $\boldsymbol{L}\left(f^{*} g^{*}\right)$ and the projection formula for unbounded complexes. With the exception of the projection formula, this was provided by Spaltenstein [27. A proof of the projection formula can be found in Lipman's notes [23], Section 3.9. He deduces it from the classical version by using homological compatibility of $\boldsymbol{R} f_{*}$ and $\underline{\lim }$.

The projection formula was used to show $\mathrm{FM}_{\mathcal{P}} \circ \mathrm{FM}_{\mathcal{P}} \cong \mathrm{FM}_{\mathcal{Q}^{\prime \bullet}}$ in the proof of Theorem 2.12. It is also used in the proof of $\mathrm{FM}_{\delta_{*} \mathcal{L}}\left(\mathcal{E}^{\bullet}\right) \cong \mathcal{E}^{\bullet} \otimes$ $\mathcal{L}$ and to show that $f^{*} \operatorname{FM}_{\mathcal{Q}^{\prime}}\left(\mathcal{E}^{\bullet}\right) \cong \operatorname{FM}_{\left(\mathbf{1}_{X} \times f\right)^{*} \mathcal{Q}^{\bullet}}\left(\mathcal{E}^{\bullet}\right)$ for unbounded complexes $\mathcal{E}^{\bullet}$ and flat morphisms $f$.

Because [23] is not yet available in final form, we confine ourselves to state the following extension of Theorem 2.12 in this remark only:

$$
\mathrm{FM}_{\mathcal{P}}^{+}: \mathrm{D}_{\text {coh }}^{+}(X) \rightarrow \mathrm{D}_{\text {coh }}^{+}(X) \text { and } \mathrm{FM}_{\mathcal{P}}: \mathrm{D}_{\text {coh }}(X) \rightarrow \mathrm{D}_{\text {coh }}(X)
$$

are equivalences of categories. The proof is the same as for the case of bounded above complexes, but is uses the results from [23].

Remark 2.14. In 4], Theorem 2.8, it is claimed that $\mathrm{FM}_{\mathcal{P}}^{\mathrm{b}}$ is an autoequivalence of the derived category $\mathrm{D}_{\text {coh }}^{\mathrm{b}}(X)$, provided $X$ is a smooth K3 surface which is elliptically fibred over $S=\mathbb{P}^{1}$. We are not able to understand all details of that proof. To be more specific, in our notation, the authors argue that the square $\mathrm{FM}_{\mathcal{Q}}^{\mathrm{b}}=\mathrm{FM}_{\mathcal{P}}^{\mathrm{b}} \circ \mathrm{FM}_{\mathcal{P}}^{\mathrm{b}}$ of the relative Fourier-Mukai functor is isomorphic to the composition of the tensor product with the shift of a certain line bundle $\mathcal{L}$ and an equivalence induced by the involution $i$ of the variety $X$. The authors show that the complex $\mathcal{Q}^{\bullet}$ has cohomology only in degree one and construct a map $f: \mathcal{H}^{1}\left(\mathcal{Q}^{\bullet}\right) \rightarrow \zeta_{*}(\mathcal{L})$, where $\zeta=\mathbf{1}_{X} \times i: X \rightarrow X \times_{S} X$. For a sheaf $\mathcal{H}$ on $X \times_{S} X$ and $z \in X \times{ }_{S} X$ let us denote its fibre over $z$ 
by $\mathcal{H}(z):=\mathcal{H} \otimes \boldsymbol{k}(z)$. In 4 it is shown that the induced map on fibres: $f(z): \mathcal{H}^{1}\left(\mathcal{Q}^{\bullet}\right)(z) \longrightarrow \zeta_{*}(\mathcal{L})(z)$ is an isomorphism for all $z \in X \times{ }_{S} X$. This would be enough to prove that $\mathcal{H}^{1}\left(\mathcal{Q}^{\bullet}\right)$ is isomorphic to $\zeta_{*}(\mathcal{L})$, if it was known that $\mathcal{H}^{1}\left(\mathcal{Q}^{\bullet}\right)$ is a sheaf of $\mathcal{O}_{\Gamma^{-}}$modules, where $\Gamma=\zeta(X)$ is the graph of $i$. However, we do not see an easy argument to show this.

\section{Fourier-Mukai transform And Grothendieck DuAlity}

In this section we study the relationship between duality and the relative Fourier-Mukai transform $\mathrm{FM}_{\mathcal{P}}$ which was studied in the previous section. We shall use Grothendieck-Verdier duality to prove our result, which describes the composition of the Fourier-Mukai transform with a functor of the type $\boldsymbol{R} \mathcal{H} \operatorname{om}(\cdot, \mathcal{L})$. A corollary of our result can be formulated as a kind of twisted compatibility with a dualising functor, but only if we assume the scheme $S$ to be Gorenstein. As a special case, we recover 12, Theorem 6.11 .

The derived functor of $\mathcal{H o m} X(\cdot, \cdot)$ is defined by replacing the second argument with an injective resolution. The result are bi-functors

$$
\begin{aligned}
& \boldsymbol{R} \mathcal{H} \operatorname{mom}_{X}(\cdot, \cdot): \mathrm{D}_{\text {coh }}^{-}(X) \times \mathrm{D}_{\text {coh }}^{+}(X) \rightarrow \mathrm{D}_{\text {coh }}^{+}(X) \quad \text { and } \\
& \boldsymbol{R} \mathcal{H} \operatorname{mom}_{X}(\cdot, \cdot): \mathrm{D}_{\text {coh }}(X) \times \mathrm{D}_{\text {coh }}^{\mathrm{b}}(X)_{\text {fid }} \rightarrow \mathrm{D}_{\text {coh }}(X),
\end{aligned}
$$

where $\mathrm{D}_{\text {coh }}^{\mathrm{b}}(X)_{\text {fid }}$ denotes the full subcategory of $\mathrm{D}_{\text {coh }}^{\mathrm{b}}(X)$ consisting of complexes with finite injective dimension, see [20], II.3.3. If $\mathcal{F}^{\bullet} \in$ $\mathrm{D}_{\text {coh }}^{-}(X), \mathcal{G}^{\bullet} \in \mathrm{D}_{\text {coh }}^{+}(X)$ and $\mathcal{L}$ is a locally free sheaf on $X$, by [20], II.5.16, we have a functorial isomorphism

$$
\boldsymbol{R} \mathcal{H} \operatorname{mom}_{X}\left(\mathcal{F}^{\bullet}, \mathcal{G}^{\bullet} \otimes \mathcal{L}\right) \cong \boldsymbol{R} \mathcal{H o m}{ }_{X}\left(\mathcal{F}^{\bullet}, \mathcal{G}^{\bullet}\right) \otimes \mathcal{L}
$$

Because we allow $X$ and $S$ to be singular, we cannot expect that the functor $\boldsymbol{R} \mathcal{H} \operatorname{mom}_{X}\left(\cdot, \mathcal{O}_{X}\right): \mathrm{D}_{\text {coh }}^{-}(X) \rightarrow \mathrm{D}_{\text {coh }}^{+}(X)$ sends $\mathrm{D}_{\text {coh }}^{\mathrm{b}}(X)$ to $\mathrm{D}_{\text {coh }}^{\mathrm{b}}(X)$. This would be true, if we supposed $S$ to be Gorenstein, but not in general. This forces us to carefully check the availability in the case of unbounded complexes of standard results like the base change theorem and the projection formula. Throughout this section, we need to use the projection formula only in the form in which it was presented in 20 .

In addition to the statements collected in Remark 2.7, which are established in [20], we need the projection formula

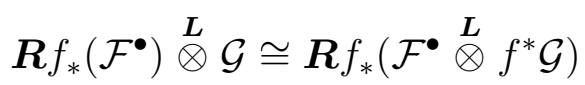

in the following situation: $f: X \rightarrow Y$ is a flat and proper morphism of Noetherian schemes of finite dimension, $\mathcal{F}^{\bullet} \in \mathrm{D}_{\text {coh }}^{+}(X)$ and $\mathcal{G}$ is a 
locally free sheaf on $Y$. In this case, $\cdot \otimes \mathcal{G}$ and $\cdot \otimes f^{*} \mathcal{G}$ are both exact. The expressions on both sides are defined by replacing $\mathcal{F}^{\bullet}$ with an injective resolution. The usual projection formula for sheaves provides the morphism and with the way-out technique from [20], I.7.1 (ii), it follows that we obtained an isomorphism.

In addition to these standard formulas, we shall need in our proof of Theorem 3.2 an isomorphism, which is provided in the following lemma.

Lemma 3.1. Let $T$ be a scheme which is of finite type over $\boldsymbol{k}$ and let $f: Y \rightarrow T$ be a projective and flat morphism whose fibres are Gorenstein curves. Suppose $\mathcal{P} \in \operatorname{Coh}(Y)$ is a T-flat sheaf, which is torsion free on fibres. Then there exists an isomorphism in $\mathrm{D}_{\text {coh }}^{+}(Y)$

$$
\boldsymbol{R} \mathcal{H o m}{ }_{Y}\left(\mathcal{P} \otimes f^{*} \mathcal{E}^{\bullet}, \mathcal{O}\right) \cong \mathcal{P}^{\vee} \otimes f^{*} \boldsymbol{R} \mathcal{H o m}{ }_{T}\left(\mathcal{E}^{\bullet}, \mathcal{O}\right)
$$

which is functorial in $\mathcal{E}^{\bullet} \in \mathrm{D}_{\text {coh }}^{-}(T)$.

Proof. The assumptions allow us to apply Corollary 2.2 to obtain flatness of $\mathcal{P}^{\vee}$ over $T$ and $\mathcal{E} x t_{Y}^{i}(\mathcal{P}, \mathcal{O})=0$ for $i>0$.

For any open subset $U \subset T$ we have a Cartesian diagram

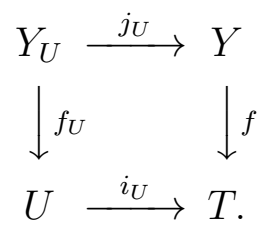

We denote $\mathcal{P}_{U}:=j_{U}^{*}(\mathcal{P})$ and observe $\mathcal{P}_{U}^{\vee} \cong j_{U}^{*}\left(\mathcal{P}^{\vee}\right)$. For any open $U \subset T$ we study the two functors

$$
\begin{aligned}
& \mathbb{A}_{U}:=\boldsymbol{R} \mathcal{H} \operatorname{Hom}_{Y_{U}}\left(\mathcal{P}_{U} \otimes f_{U}^{*}(\cdot), \mathcal{O}_{Y_{U}}\right) \quad \text { and } \\
& \mathbb{B}_{U}:=\mathcal{P}_{U}^{\vee} \otimes f_{U}^{*} \boldsymbol{R} \mathcal{H o m}\left(\cdot, \mathcal{O}_{U}\right) .
\end{aligned}
$$

Because $\mathcal{P}$ is supposed to be $T$-flat, $\mathcal{P}_{U}$ is $U$-flat and the functor $\mathcal{P}_{U} \otimes$ $f_{U}^{*}(\cdot)$ is exact. From [20, I $\S 7$, it is, therefore, clear that both functors $\mathbb{A}_{U}$ and $\mathbb{B}_{U}$ are contravariant way-out right $\partial$-functors. Because the sheaf $\mathcal{E} x t^{i}(\mathcal{F}, \mathcal{G})$ is coherent for any $i$ and arbitrary coherent sheaves $\mathcal{F}, \mathcal{G}$, it follows from 20], II.7.3, that we have

$$
\mathbb{A}_{U}, \mathbb{B}_{U}: \mathrm{D}_{\text {coh }}^{-}(U) \rightarrow \mathrm{D}_{\text {coh }}^{+}\left(Y_{U}\right) .
$$

Our main aim is to show $\mathbb{A}_{T} \cong \mathbb{B}_{T}$. We are going to apply the lemma on way-out functors [20, Prop. I.7.1. This requires that we have welldefined natural transformations $\eta_{U}: \mathbb{A}_{U} \rightarrow \mathbb{B}_{U}$ for any open $U \subset T$ and a compatibility between them. For any $\mathcal{E}^{\bullet} \in \mathrm{D}_{\text {coh }}^{-}(U)$ we define

$$
\eta_{U}\left(\mathcal{E}^{\bullet}\right): \mathbb{A}_{U}\left(\mathcal{E}^{\bullet}\right) \rightarrow \mathbb{B}_{U}\left(\mathcal{E}^{\bullet}\right)
$$


as follows. We choose a resolution by injective quasi-coherent sheaves $\mathcal{O}_{U} \rightarrow \mathcal{I}_{U}^{\bullet}$. Because $f_{U}$ is flat, $\mathcal{O}_{Y_{U}} \cong f_{U}^{*} \mathcal{O}_{U} \rightarrow f_{u}^{*} \mathcal{I}_{U}^{\bullet}$ is still a quasiisomorphism. Let now $\mathcal{O}_{Y_{U}} \rightarrow \mathcal{J}_{U}^{\bullet}$ be an injective resolution. The Comparison Theorem (see e.g. [29], 2.3.7) implies that $\mathcal{O}_{Y_{U}} \rightarrow \mathcal{J}_{U}^{\bullet}$ factors through $f_{U}^{*} \mathcal{O}_{U} \rightarrow f_{u}^{*} \mathcal{I}_{U}^{\bullet}$, so that we obtain two quasi-isomorphisms

$$
\mathcal{O}_{Y_{U}} \rightarrow f_{U}^{*} \mathcal{I}_{U}^{\bullet} \rightarrow \mathcal{J}_{U}^{\bullet}
$$

By the construction of derived functors, there are natural isomorphisms $\mathbb{A}_{U}\left(\mathcal{E}^{\bullet}\right) \cong \mathcal{P}_{U}^{\vee} \otimes f_{U}^{*} \mathcal{H} \operatorname{lom}_{U}\left(\mathcal{E}^{\bullet}, \mathcal{I}_{U}^{\bullet}\right)$ and $\mathbb{B}_{U}\left(\mathcal{E}^{\bullet}\right) \cong \mathcal{H} o m_{Y_{U}}\left(\mathcal{P}_{U} \otimes\right.$ $f_{U}^{*} \mathcal{E}^{\bullet}, \mathcal{J}_{U}^{\bullet}$ ), such that the choice of a different resolution leads to the isomorphism which is obtained by composing the above with the map which is induced by the unique homotopy equivalence between the resolutions. We define $\eta_{U}\left(\mathcal{E}^{\bullet}\right)$ to be the composition of these isomorphisms with the following morphisms:

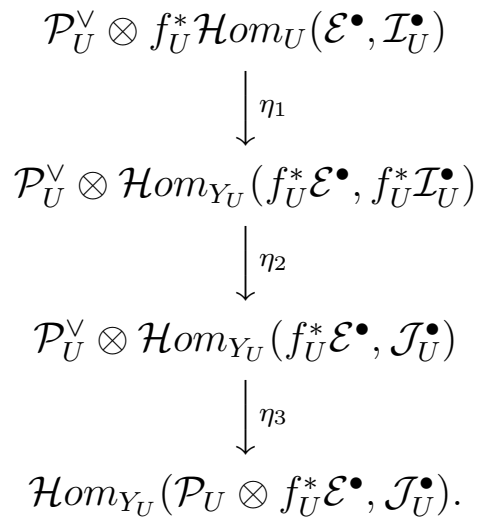

The morphism $\eta_{2}$ is induced by the quasi-isomorphism $f_{U}^{*} \mathcal{I}_{U}^{\bullet} \rightarrow \mathcal{J}_{U}^{\bullet}$. The morphisms $\eta_{1}$ and $\eta_{3}$ are the canonical ones, see for example 19, $0_{\text {I }}$ 4.4.6 and 5.4.2. All these maps are natural with respect to $\mathcal{E}^{\bullet} \in$ $\mathrm{D}_{\text {coh }}^{-}(U)$. Although, $\eta_{1}, \eta_{2}$ and $\eta_{3}$ are homomorphisms of complexes of $\mathcal{O}_{Y_{U}}$-modules, we are interested in $\eta_{U}\left(\mathcal{E}^{\bullet}\right)$ as a morphism in $\mathrm{D}_{\text {coh }}^{+}\left(Y_{U}\right)$ only. As such, it does not depend on the choice of the resolutions $\mathcal{I}_{U}^{\bullet}$ and $\mathcal{J}_{U}^{\bullet}$. This follows from the Comparison Theorem and the fact that homotopy equivalent morphisms coincide as morphisms in the derived category.

An important tool in our proof will be the following compatibility. If $U \subset T$ is an open subset, there exist isomorphisms of functors $\mathrm{D}_{\text {coh }}^{-}(T) \rightarrow \mathrm{D}_{\text {coh }}^{+}\left(Y_{U}\right)$

$$
\mathbb{A}_{U} \circ i_{U}^{*} \cong j_{U}^{*} \circ \mathbb{A}_{T} \quad \text { and } \quad \mathbb{B}_{U} \circ i_{U}^{*} \cong j_{U}^{*} \circ \mathbb{B}_{T}
$$


such that the diagram

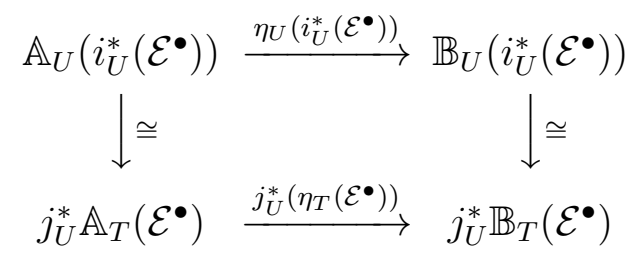

is commutative. This follows from our definition, because $j_{U}^{*} \circ f_{U}^{*}=$ $f_{U}^{*} \circ i_{U}^{*}$, as well as $i_{U}^{*} \mathcal{H} o m_{T}\left(\mathcal{F}^{\bullet}, \mathcal{G}^{\bullet}\right)=\mathcal{H o m}_{U}\left(i_{U}^{*} \mathcal{F}^{\bullet}, i_{U}^{*} \mathcal{G}^{\bullet}\right)$ and because the definition of $\eta$ does not depend on the choice of resolutions, as observed above.

Next, we prove for any open $U \subset T$ that $\eta_{U}\left(\mathcal{O}_{U}\right)$ is an isomorphism in $\mathrm{D}_{\text {coh }}^{+}\left(Y_{U}\right)$. Because $\mathcal{E} x t_{U}^{i}\left(\mathcal{O}_{U}, \mathcal{O}_{U}\right)=0$ for all $i>0$, the complex $\boldsymbol{R} \mathcal{H}$ om $_{U}\left(\mathcal{O}_{U}, \mathcal{O}_{U}\right)$ is isomorphic to the sheaf $\mathcal{O}_{U}$ concentrated in degree zero. Hence, $\mathbb{A}_{U}\left(\mathcal{O}_{U}\right) \cong \mathcal{P}_{U}^{\vee}$. On the other hand, $\mathcal{E} x t_{Y}^{i}\left(\mathcal{P}, \mathcal{O}_{Y}\right)=0$ for all $i>0$ implies $\mathbb{B}_{U}\left(\mathcal{O}_{U}\right) \cong \mathcal{H} o m\left(\mathcal{P}_{U}, \mathcal{O}_{U}\right)=\mathcal{P}_{U}^{\vee}$. By definition, $\eta_{U}\left(\mathcal{O}_{U}\right)$ is the composition of the top row in the commutative diagram

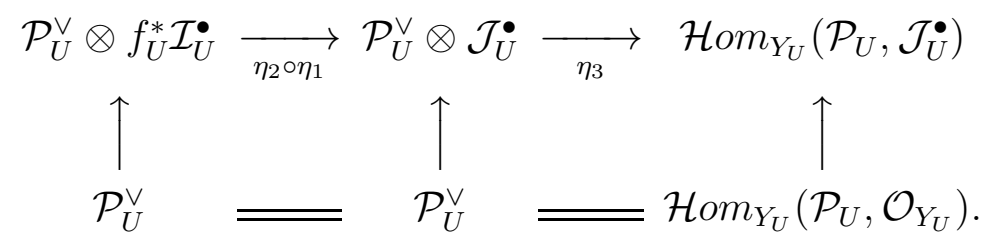

Because the functor $\mathcal{P}_{U}^{\vee} \otimes f_{U}^{*}(\cdot)$ is exact, the first vertical arrow is a quasi-isomorphism. The third vertical arrow is a quasi-isomorphism as well, because $\mathcal{E} x t_{Y}^{i}\left(\mathcal{P}, \mathcal{O}_{Y}\right)=0$ for all $i>0$. Hence, $\eta_{U}\left(\mathcal{O}_{U}\right)$ is a quasi-isomorphism. Because $\mathbb{A}_{U}, \mathbb{B}_{U}$ and $\eta_{U}$ are compatible with finite direct sums, $\eta_{U}\left(\mathcal{O}_{U}^{\oplus k}\right)$ is an isomorphism in $\mathrm{D}_{\text {coh }}^{+}\left(Y_{U}\right)$ for all $k \geq 1$.

If $\mathcal{E}$ is now an arbitrary locally free sheaf of finite rank on $T$, using diagram (14) we obtain that there exists an open covering of $T$ such that $j_{U}^{*}\left(\eta_{T}(\mathcal{E})\right)$ is an isomorphism for any open set $U \subset T$ in this covering. This implies that $\eta_{T}(\mathcal{E})$ is an isomorphism. The same statement is true for $\eta_{U}(\mathcal{E})$ if $\mathcal{E}$ is a locally free coherent sheaf on an arbitrary open subset $U \subset T$. Because on any quasi-projective open subset $U \subset T$, any coherent sheaf has a resolution by coherent locally free sheaves, [20], Prop. I.7.1 (iv), implies that $\eta_{U}(\mathcal{E})$ is an isomorphism if $\mathcal{E}$ is an arbitrary coherent sheaf on a quasi-projective open subset $U \subset T$.

We assumed $T$ to be of finite type over $\boldsymbol{k}$. This implies that there exists an open affine cover of $T$ by quasi-projective schemes. As before, using the diagram (14), we obtain that $\eta_{T}(\mathcal{E})$ is an isomorphism for any coherent sheaf $\mathcal{E}$ on $T$. The claim follows now from the lemma on wayout functors [20], I.7.1. 
Grothendieck-Verdier duality can be formulated in the following way (see [20, 15]): if $f: X \rightarrow Y$ is a proper morphism between schemes which are of finite type over $\boldsymbol{k}$, there exists a functor

$$
f^{!}: \mathrm{D}_{\text {coh }}^{+}(Y) \rightarrow \mathrm{D}_{\text {coh }}^{+}(X),
$$

such that there is an isomorphism

$$
\boldsymbol{R} f_{*} \boldsymbol{R} \mathcal{H o m}{ }_{X}\left(\mathcal{F}^{\bullet}, f^{!} \mathcal{G}^{\bullet}\right) \cong \boldsymbol{R} \mathcal{H o m}{ }_{Y}\left(\boldsymbol{R} f_{*} \mathcal{F}^{\bullet}, \mathcal{G}^{\bullet}\right)
$$

which is functorial in both arguments $\mathcal{F}^{\bullet} \in \mathrm{D}_{\mathrm{qc}}^{-}(X)$ and $\mathcal{G}^{\bullet} \in \mathrm{D}_{\text {coh }}^{+}(Y)$. On the other hand, an object $\mathcal{I}^{\bullet} \in \mathrm{D}_{\text {coh }}^{\mathrm{b}}(Y)$ of finite injective dimension is called a dualising complex, if the functor $\mathbb{D}_{Y}:=\boldsymbol{R} \mathcal{H o m}_{Y}\left(\cdot, \mathcal{I}^{\bullet}\right)$ satisfies $\mathbb{D}_{Y} \circ \mathbb{D}_{Y} \cong \mathbf{1}$ on the category $\mathrm{D}_{\text {coh }}(Y)$. Such a dualising complex exists on any scheme $Y$ which is of finite type over $\boldsymbol{k}$. An important feature of the functor $f^{!}$is that it respects the property of a complex to be dualising. Hence, the functor $\mathbb{D}_{X}:=\boldsymbol{R} \mathcal{H} \operatorname{com}_{X}\left(\cdot, f^{!} \mathcal{I}^{\bullet}\right)$ is dualising and the duality theorem implies

$$
\boldsymbol{R} f_{*} \circ \mathbb{D}_{X} \cong \mathbb{D}_{Y} \circ \boldsymbol{R} f_{*}
$$

If $\mathcal{I}^{\bullet}$ is a dualising complex on $Y$, for any integer $n$ and any invertible sheaf $\mathcal{L}$ on $Y$, the complex $\mathcal{I}^{\bullet}[n] \otimes \mathcal{L}$ is a dualising complex as well. Up to such changes, dualising complexes are unique. The notation $\mathbb{D}_{Y}$ must be used with care, because such a functor depends on the choice of a dualising complex on $Y$, but only up to a shift and a twist by an invertible sheaf.

In order to apply the duality theorem, it is important to be able to calculate $f^{!}$. This is particularly easy in the special case of a CohenMacaulay morphism. If $Y$ is of finite type over $\boldsymbol{k}$ and $f: X \rightarrow Y$ is a projective Cohen-Macaulay morphism whose fibres are of pure dimension $n$, we have for any $\mathcal{F}^{\bullet} \in \mathrm{D}_{\text {coh }}^{+}(Y)$ by [15], Theorem 4.3.2:

$$
f^{!} \mathcal{F}^{\bullet} \cong \omega_{f}[n] \stackrel{\boldsymbol{L}}{\otimes} f^{*} \mathcal{F}^{\bullet} .
$$

Here we denoted by $\omega_{f}$ the relative dualising sheaf, which is a coherent $Y$-flat sheaf on $X$ and which satisfies $\omega_{f}[n] \cong f^{!} \mathcal{O}_{Y}$, see [15], Theorem 3.5.1. This sheaf is locally free if and only if the morphism $f$ is Gorenstein. The sheaf $\omega_{f}$ is compatible with base-change ([15], Theorem 3.6.1). This implies that its restriction to fibres is the dualising sheaf on the fibre. In particular, if $f$ is a proper Gorenstein morphism all fibres of which have trivial dualising sheaf and if $Y$ is reduced, then

$$
\omega_{f} \cong f^{*} \mathcal{A}
$$

with an invertible sheaf $\mathcal{A}$ on $Y$. 
In the formulation of the following theorem we use notation from Section 2. If $q: X \rightarrow S$ satisfies the assumptions $(\star)$, there exists an invertible sheaf $\mathcal{A}$ on $S$ such that $\omega_{q} \cong q^{*} \mathcal{A}$. The sheaf $\mathcal{P}$ on $X \times{ }_{S} X$ was defined in (11) and $\mathcal{M}$ is the sheaf introduced in Proposition 2.3. which satisfies $\left(\mathbf{1}_{X} \times i\right)^{*} \mathcal{P} \cong \mathcal{P}^{\vee} \otimes \pi^{*} \mathcal{M}$. Finally, $\mathcal{L}$ denotes an invertible sheaf on $S$. Note that the functor $\boldsymbol{R} \mathcal{H} \operatorname{mom}_{X}\left(\cdot, q^{*} \mathcal{L}\right)$ is a dualising functor on $X$ only if $X$ is Gorenstein. This case is considered in Corollary 3.3.

Theorem 3.2. Suppose $S, X$ and $q: X \rightarrow S$ satisfy condition $(\star)$ from Section [2. Then, there exists an isomorphism in $\mathrm{D}_{\text {coh }}^{+}(X)$, which is functorial in $\mathcal{E}^{\bullet} \in \mathrm{D}_{\text {coh }}^{-}(X)$ :

$\boldsymbol{R} \mathcal{H} o m_{X}\left(\mathrm{FM}_{\mathcal{P}}^{-}\left(\mathcal{E}^{\bullet}\right), q^{*} \mathcal{L}\right) \cong i^{*} \operatorname{FM}_{\mathcal{P}}^{+}\left(\boldsymbol{R} \mathcal{H} o m_{X}\left(\mathcal{E}^{\bullet}, q^{*} \mathcal{L}\right)\right) \otimes q^{*}\left(\mathcal{M}^{\vee} \otimes \mathcal{A}\right)[1]$

Proof. By definition, we have

$$
\boldsymbol{R} \mathcal{H o m}{ }_{X}\left(\mathrm{FM}_{\mathcal{P}}^{-}\left(\mathcal{E}^{\bullet}\right), q^{*} \mathcal{L}\right) \cong \boldsymbol{R} \mathcal{H} \operatorname{mom}_{X}\left(\boldsymbol{R} \pi_{2 *}\left(\mathcal{P} \otimes \pi_{1}^{*} \mathcal{E}^{\bullet}\right), q^{*} \mathcal{L}\right)
$$

and apply Grothendieck-Verdier duality to obtain

$$
\cong \boldsymbol{R} \pi_{2 *} \boldsymbol{R} \mathcal{H} \operatorname{Hom}_{X{ }_{S} X}\left(\mathcal{P} \otimes \pi_{1}^{*} \mathcal{E}^{\bullet}, \pi_{2}^{!} q^{*} \mathcal{L}\right)
$$

Using [15], Theorem 3.6.1, we see

$$
\pi_{2}^{!} q^{*} \mathcal{L} \cong \omega_{\pi_{2}}[1] \otimes \pi_{2}^{*} q^{*} \mathcal{L} \cong \pi_{1}^{*} \omega_{q}[1] \otimes \pi_{2}^{*} q^{*} \mathcal{L} \cong \pi^{*}(\mathcal{A} \otimes \mathcal{L})[1]
$$

Therefore, the above is isomorphic to

$$
\boldsymbol{R} \pi_{2 *}\left(\boldsymbol{R} \mathcal{H o m}{ }_{X{ }_{S} X}\left(\mathcal{P} \otimes \pi_{1}^{*} \mathcal{E}^{\bullet}, \mathcal{O}_{X \times_{S} X}\right) \otimes \pi^{*}(\mathcal{A} \otimes \mathcal{L})[1]\right) .
$$

Applying Lemma 3.1 and using $\pi^{*} \mathcal{L} \cong \pi_{1}^{*} q^{*} \mathcal{L}$, we see that this is functorially isomorphic to

$$
\boldsymbol{R} \pi_{2 *}\left(\mathcal{P}^{\vee} \otimes \pi_{1}^{*} \boldsymbol{R} \mathcal{H} \operatorname{mom}_{X}\left(\mathcal{E}^{\bullet}, q^{*} \mathcal{L}\right) \otimes \pi^{*} \mathcal{A}\right)[1] .
$$

Now we use $\pi^{*} \mathcal{A} \cong \pi_{2}^{*} q^{*} \mathcal{A}, \pi_{1} \circ\left(\mathbf{1}_{X} \times i\right)=\pi_{1}$ and $\mathcal{P}^{\vee} \cong\left(\mathbf{1}_{X} \times\right.$ $i)^{*} \mathcal{P} \otimes \pi^{*} \mathcal{M}^{\vee}$ with $\mathcal{M}$ being the invertible sheaf on $S$ introduced in Proposition 2.3. This allows us to write the above expression as

$$
\boldsymbol{R} \pi_{2 *}\left(\left(\mathbf{1}_{X} \times i\right)^{*} \mathcal{P} \otimes\left(\mathbf{1}_{X} \times i\right)^{*} \pi_{1}^{*} \boldsymbol{R} \mathcal{H} \operatorname{mom}_{X}\left(\mathcal{E}^{\bullet}, q^{*} \mathcal{L}\right) \otimes \pi_{2}^{*} q^{*}\left(\mathcal{M}^{\vee} \otimes \mathcal{A}\right)\right)[1]
$$

Finally, we apply the projection formula, followed by $\boldsymbol{R} \pi_{2 *} \circ\left(\mathbf{1}_{X} \times i\right)^{*} \cong$ $i^{*} \circ \boldsymbol{R} \pi_{2 *}$, which is base-change with the isomorphism $i$, and obtain the desired isomorphism

$$
\begin{gathered}
\boldsymbol{R} \pi_{2 *}\left(\left(\mathbf{1}_{X} \times i\right)^{*}\left(\mathcal{P} \otimes \pi_{1}^{*} \boldsymbol{R} \mathcal{H} \operatorname{Hom}_{X}\left(\mathcal{E}^{\bullet}, q^{*} \mathcal{L}\right)\right)\right) \otimes q^{*}\left(\mathcal{M}^{\vee} \otimes \mathcal{A}\right)[1] \\
\cong i^{*} \operatorname{FM}_{\mathcal{P}}^{+}\left(\boldsymbol{R H} \operatorname{Hom}_{X}\left(\mathcal{E}^{\bullet}, q^{*} \mathcal{L}\right)\right) \otimes q^{*}\left(\mathcal{M}^{\vee} \otimes \mathcal{A}\right)[1]
\end{gathered}
$$


Corollary 3.3. In addition to the notation and assumptions of Theorem [3.2, suppose $S$ is Gorenstein and connected. Then, there exist isomorphism

$$
\begin{aligned}
& \mathbb{D}_{X} \circ \mathrm{FM}_{\mathcal{P}}^{-} \cong[1] \circ \mathbb{T}_{\mathcal{M}^{\vee} \otimes \mathcal{A}} \circ i^{*} \circ \mathrm{FM}_{\mathcal{P}}^{+} \circ \mathbb{D}_{X} \text { and } \\
& \mathbb{D}_{X} \circ \mathrm{FM}_{\mathcal{P}}^{\mathrm{b}} \cong[1] \circ \mathbb{T}_{\mathcal{M}^{\vee} \otimes \mathcal{A}} \circ i^{*} \circ \mathrm{FM}_{\mathcal{P}}^{\mathrm{b}} \circ \mathbb{D}_{X},
\end{aligned}
$$

where $\mathbb{T}_{\mathcal{M}^{\vee} \otimes \mathcal{A}}$ denotes the tensor product functor with the locally free sheaf $q^{*}\left(\mathcal{M}^{\vee} \otimes \mathcal{A}\right)$ and $\mathbb{D}_{X}$ is a dualising functor on $X$ of the form $\boldsymbol{R} \mathcal{H} \operatorname{mom}_{X}\left(-, q^{*} \mathcal{L}\right)$, with an invertible sheaf $\mathcal{L}$ on $S$.

Proof. Because the scheme $S$ and the morphism $q$ are Gorenstein, the scheme $X$ is Gorenstein as well. Hence, any shift of a locally free sheaf on $X$ is a dualising complex. In particular, for any locally free sheaf $\mathcal{L}$ on $S$, the functor $\boldsymbol{R} \mathcal{H o m}{ }_{X}\left(-, q^{*} \mathcal{L}\right)$ is dualising on $X$. The claim is just a reformulation of the theorem. The statement involving $\mathrm{FM}_{\mathcal{P}}^{\mathrm{b}}$ is obtained by restricting the other one, because $X$ is Gorenstein, hence $\mathbb{D}_{X}: \mathrm{D}_{\text {coh }}^{\mathrm{b}}(X) \rightarrow \mathrm{D}_{\text {coh }}^{\mathrm{b}}(X)$.

In the special case $S=\operatorname{Spec}(\boldsymbol{k})$, the invertible sheaves $\mathcal{M}$ and $\mathcal{A}$ are automatically trivial, so that $\mathbb{T}_{\mathcal{M}^{\vee} \otimes \mathcal{A}} \cong 1$. Because any irreducible projective curve of arithmetic genus one is automatically Gorenstein with trivial canonical sheaf, we obtain the following corollary, which generalises Mukai's result [25], (3.8). We denote $\mathbb{D}_{\boldsymbol{E}}=\boldsymbol{R} \mathcal{H} \operatorname{Hom}_{\boldsymbol{E}}\left(-, \mathcal{O}_{\boldsymbol{E}}\right)$.

Corollary 3.4. If $\boldsymbol{E}$ is an irreducible projective curve of arithmetic genus one, then:

$$
\begin{aligned}
& \mathbb{D}_{\boldsymbol{E}} \circ \mathrm{FM}_{\mathcal{P}}^{-} \cong[1] \circ i^{*} \circ \mathrm{FM}_{\mathcal{P}}^{+} \circ \mathbb{D}_{\boldsymbol{E}} \quad \text { and } \\
& \mathbb{D}_{\boldsymbol{E}} \circ \mathrm{FM}_{\mathcal{P}}^{\mathrm{b}} \cong[1] \circ i^{*} \circ \mathrm{FM}_{\mathcal{P}}^{\mathrm{b}} \circ \mathbb{D}_{\boldsymbol{E}} .
\end{aligned}
$$

This is a generalisation of 12, Theorem 6.11. To see this, recall that we studied in [12] the functor $\mathbb{F}:=[1] \circ \mathrm{FM}_{\mathcal{P}}^{\mathrm{b}}$. Moreover, for any coherent torsion sheaf $\mathcal{F}$ we called $\mathbb{M}(\mathcal{F})=\mathcal{E} x t^{1}(\mathcal{F}, \mathcal{O})$ the Matlis dual of $\mathcal{F}$. Note that we have $\mathcal{E} x t^{i}(\mathcal{F}, \mathcal{O})=0$ for such $\mathcal{F}$ and $i \neq 1$. This implies $\mathbb{D}_{E}(\mathcal{F}) \cong \mathbb{M}(\mathcal{F})[-1]$. For any semi-stable torsion free sheaf $\mathcal{E}$ of degree zero on $\boldsymbol{E}$ we have shown in [12, Theorem 6.11, that there is an isomorphism $\mathbb{M}(\mathbb{F}(\mathcal{E})) \cong i^{*} \mathbb{F}\left(\mathcal{E}^{\vee}\right)$. Now, observe that we have $\mathcal{E} x t^{i}(\mathcal{E}, \mathcal{O})=0$ for any torsion free sheaf $\mathcal{E}$ on $\boldsymbol{E}$ and all $i>0$. Hence, $\mathbb{D}_{\boldsymbol{E}}(\mathcal{E}) \cong \mathcal{E}^{\vee}$. Corollary 3.4 together with $\mathbb{D}_{\boldsymbol{E}} \circ[-1] \cong[1] \circ \mathbb{D}_{\boldsymbol{E}}$ gives, therefore,

$$
\begin{gathered}
\mathbb{M}(\mathbb{F}(\mathcal{E})) \cong \mathbb{D}_{\boldsymbol{E}}(\mathbb{F}(\mathcal{E}))[1] \cong \mathbb{D}_{\boldsymbol{E}}\left(\mathrm{FM}_{\mathcal{P}}^{\mathrm{b}}(\mathcal{E})\right) \cong i^{*} \mathrm{FM}_{\mathcal{P}}^{\mathrm{b}}\left(\mathbb{D}_{\boldsymbol{E}}(\mathcal{E})\right)[1] \\
\cong i^{*} \mathbb{F}\left(\mathbb{D}_{\boldsymbol{E}}(\mathcal{E})\right) \cong i^{*} \mathbb{F}\left(\mathcal{E}^{\vee}\right)
\end{gathered}
$$




\section{Applications}

The purpose of this section is to show through simple examples how the results of the previous sections can be used to explicitly construct interesting families of sheaves. In order to be able to study sheaves on the fibres, we need the following compatibility of Fourier-Mukai transforms with restriction functors.

Lemma 4.1. Suppose $q: X \rightarrow S$ satisfies condition (*) from Section 2. let $s: \operatorname{Spec}(\boldsymbol{k}) \rightarrow S$ be an arbitrary point and denote by $i_{s}: X_{s} \rightarrow X$ the embedding of the fibre of $q$ over $s$. Let $\mathcal{P}$ be the sheaf (1) on $X \times_{S} X$. $B y \mathcal{P}_{s}$ we denote its restriction to $X_{s} \times X_{s}$. If $\mathcal{E}$ is an $S$-flat coherent sheaf on $X$, there exists an isomorphism

$$
\boldsymbol{L} i_{s}^{*} \mathrm{FM}_{\mathcal{P}}^{\mathrm{b}}(\mathcal{E}) \cong \mathrm{FM}_{\mathcal{P}_{s}}^{\mathrm{b}} i_{s}^{*}(\mathcal{E})
$$

Moreover, if $\mathrm{FM}_{\mathcal{P}_{s}}^{\mathrm{b}} i_{s}^{*}(\mathcal{E})$ is a sheaf for all $s \in S, \mathrm{FM}_{\mathcal{P}}^{\mathrm{b}}(\mathcal{E})$ is an $S$-flat sheaf on $X$ and we can replace $\boldsymbol{L} i_{s}^{*}$ by $i_{s}^{*}$ in this formula.

Proof. Because $\mathcal{E}$ and $\mathcal{P}$ are both $S$-flat, the sheaf $\mathcal{P} \otimes \pi_{1}^{*} \mathcal{E}$ is $\pi_{2}$-flat as well. Hence, we have a base-change isomorphism (see [24], II §5)

$$
\boldsymbol{L} i_{s}^{*} \boldsymbol{R} \pi_{2 *}\left(\mathcal{P} \otimes \pi_{1}^{*} \mathcal{E}\right) \cong \boldsymbol{R} p_{2 *}\left(i_{s} \times i_{s}\right)^{*}\left(\mathcal{P} \otimes \pi_{1}^{*} \mathcal{E}\right),
$$

which implies (15). The final statement follows from [8], Lemma 4.3.

We conclude this article by presenting two examples of fibrewise semi-stable torsion free sheaves on an elliptic fibration $X \rightarrow S$. The main idea of the construction is to apply the relative Fourier-Mukai transform $\mathrm{FM}_{\mathcal{P}}^{\mathrm{b}}$ to an $S$-flat family of torsion sheaves on $X$. The result is a flat family of semi-stable torsion free sheaves. This is a special case of the spectral cover construction [17, 16]. Moreover, using our previous results about Fourier-Mukai transforms on Weierstraß cubics 12, we are able to describe explicitly the restriction onto the singular fiber in terns of étale coverings of nodal cubic curves.

Let $X \subset \mathbb{P}^{2} \times \mathbb{A}^{1}$ be the family of irreducible cubics, defined by the polynomial

$$
F_{t}(x, y, z)=y^{2} z-x^{3}-x^{2} z-t(1-t) x z^{2}+t^{2} z^{3},
$$

where $(x: y: z)$ are homogeneous coordinates on $\mathbb{P}^{2}$ and $t$ is an affine coordinate on $\mathbb{A}^{1}$. The discriminant of this family is a polynomial in $t$ of degree six which vanishes at $t=0$. The general fibre of the projection $X \rightarrow \mathbb{A}^{1}$ is smooth. The fibre $X_{0}$ over $t=0$ is given by the polynomial $y^{2} z-x^{3}-x^{2} z$, hence is an irreducible cubic with a node at $(x: y: z)=(0: 0: 1)$. This family has a section $\sigma: \mathbb{A}^{1} \rightarrow X$, which is given by $\sigma(t)=((0: 1: 0), t) \in X$. 
Example 4.2. For any $\lambda \in \boldsymbol{k}^{\times}$, we denote by $C_{\lambda}$ the intersection of $X$ with the surface which is given in $\mathbb{P}^{2} \times \mathbb{A}^{1}$ by the equation

$$
(1+\lambda) y-(1-\lambda) x=0 .
$$

We first study the case $\lambda \neq-1$. In this case, $C_{\lambda}$ is disjoint to the set where $z=0$. Therefore, it is sufficient to work on the affine set $z \neq 0$, where the curve $C_{\lambda}$ is defined by the ideal

$$
I_{\lambda}=\left\langle(1+\lambda) y-(1-\lambda) x, y^{2}-x^{3}-x^{2}-t(1-t) x+t^{2}\right\rangle .
$$

If $t \in \boldsymbol{k}$ is arbitrary, the module $\boldsymbol{k}[x, y] / I_{\lambda}$ is of length three. For generic $t$ it consists of three simple points. Note that the sheaf $\mathcal{O}_{C_{\lambda}}$ is flat over $\mathbb{A}^{1}$, because $t$ is not a zero-divisor of $\boldsymbol{k}[x, y, t] / I_{\lambda}$.

The fibre of $\mathcal{O}_{C_{\lambda}}$ over $t=0$ is given by the ideal

$$
\begin{aligned}
& \left\langle(1+\lambda) y-(1-\lambda) x, y^{2}-x^{3}-x^{2}\right\rangle \\
= & \left\langle(1+\lambda) y-(1-\lambda) x, x^{2}\left(\frac{4 \lambda}{(1+\lambda)^{2}}+x\right)\right\rangle \subset \boldsymbol{k}[x, y] .
\end{aligned}
$$

Thus, the support of $i_{0}^{*} \mathcal{O}_{C_{\lambda}}$ consists of the two points $(0: 0: 1)$ and $\left(4 \lambda(1+\lambda): 4 \lambda(1-\lambda):-(1+\lambda)^{3}\right)$ in $\mathbb{P}^{2}$. At the second of them, $i_{0}^{*} \mathcal{O}_{C_{\lambda}}$ is of length one. In order to understand $i_{0}^{*} \mathcal{O}_{C_{\lambda}}$ at the singular point, we look at the completion $\widehat{M}$ at the point $(x, y)=(0,0)$ of

$$
M=\boldsymbol{k}[x, y] /\left\langle(1+\lambda) y-(1-\lambda) x, x^{2}\left(4 \lambda+(1+\lambda)^{2} x\right)\right\rangle .
$$

As we always assume $\lambda \neq 0$, the factor behind $x$ is a unit in $\widehat{R}=$ $\boldsymbol{k}[[x, y]] /\left\langle y^{2}-x^{2}-x^{3}\right\rangle$, hence $\widehat{M}=\boldsymbol{k}[[x, y]] /\left\langle(1+\lambda) y-(1-\lambda) x, x^{2}\right\rangle$. If we let $\widetilde{x}:=x \sqrt{1+x} \in \widehat{R}$ and define $\xi=\widetilde{x}-y, \eta=\widetilde{x}+y$, we obtain an isomorphism $\widehat{R} \cong \boldsymbol{k}[[\xi, \eta]] /\langle\xi \eta\rangle$.

Note that $\sqrt{1+x}=1+x / 2-x^{2} / 8+\ldots$, as given by the binomial series, is a unit in $\widehat{R}$. Because $\widetilde{x}-x \in\left\langle x^{2}\right\rangle \subseteq \widehat{R}$ and $x^{2}=0$ in $\widehat{M}$, we obtain in $\widehat{M}$

$$
\lambda \eta-\xi=\lambda(y+\widetilde{x})+y-\widetilde{x}=(1+\lambda) y-(1-\lambda) x=0 .
$$

This implies $\widehat{M} \cong \widehat{R} /\langle\lambda \eta-\xi\rangle$. Using the notation from [12], $\S 4$, this module is identified with the band module $\mathcal{M}((1,1), 1, \lambda)$ in the classification of Gelfand and Ponomarev. It has length two, as expected.

The functor $\mathbb{F}=[1] \circ \mathrm{FM}_{\mathcal{P}_{0}}^{\mathrm{b}}$ was shown in $\left[12\right.$ to satisfy $\mathbb{F} \circ \mathbb{F} \cong i^{*}[1]$ and $\mathbb{F}(\mathcal{B}((1,-1), 1, \lambda)) \cong \mathcal{M}((1,1), 1, \lambda)$. Here, $i$ is an involution on $X_{0}$ and $\mathcal{B}((1,-1), 1, \lambda)$ a semi-stable indecomposable vector bundle of rank two and degree zero on $X_{0}$. This implies

$$
\mathrm{FM}_{\mathcal{P}_{0}}^{\mathrm{b}}\left(i_{0}^{*} \mathcal{O}_{C_{\lambda}}\right) \cong \mathcal{B}((1,-1), 1, \lambda) \oplus \mathcal{L}_{\lambda}
$$

where $\mathcal{L}_{\lambda}$ is a line bundle on $X_{0}$ which depends on $\lambda$. 
As we have seen above, for generic $t \in \mathbb{A}^{1}$ the fibre of $C_{\lambda}$ over $t$ consists of three points of length one. This implies that $\operatorname{FM}_{\mathcal{P}_{t}}^{\mathrm{b}}\left(i_{t}^{*} \mathcal{O}_{C_{\lambda}}\right)$ is the direct sum of three line bundles. Using Lemma 4.1, we obtain that $\operatorname{FM}_{\mathcal{P}}^{\mathrm{b}}\left(\mathcal{O}_{C_{\lambda}}\right)$ is a coherent $\mathbb{A}^{1}$-flat sheaf on $X$, which is the direct sum of three line bundles on the generic fibre, but which has a direct summand isomorphic to the indecomposable vector bundle $\mathcal{B}((1,-1), 1, \lambda)$ if restricted to the singular fibre $X_{0}$.

In the case $\lambda=-1$, the situation is very similar. The main difference is that the image of the section $\sigma$ is a component of $C_{-1}$. On the affine open set $z \neq 0$, we obtain $I_{-1}=\left\langle x, y^{2}+t^{2}\right\rangle$ and, for any fixed $t \in \boldsymbol{k}$, the module $\boldsymbol{k}[x, y] / I_{-1}$ has length two. This module consists of two simple points for all $t \neq 0$. Hence, the component of $C_{-1}$, which is not supported at the image of the section $\sigma$, is of degree two over $\mathbb{A}^{1}$. The restriction of this component to the fibre $X_{0}$ is supported at the singular point. Its completion is isomorphic to $\widehat{R} /\langle\xi+\eta\rangle$. This is the band module $\mathcal{M}((1,1), 1,-1)$ about which we know $\mathbb{F}(\mathcal{B}((1,-1), 1,-1)) \cong$ $\mathcal{M}((1,1), 1,-1)$.

Using Lemma 4.1, this implies that $\mathrm{FM}_{\mathcal{P}}^{\mathrm{b}}\left(\mathcal{O}_{C_{-1}}\right)$ splits into a direct sum of two $\mathbb{A}^{1}$-flat families $\mathcal{A}_{1} \oplus \mathcal{A}_{2}$. The restriction of $\mathcal{A}_{1}$ on each fibre is trivial. However, $\mathcal{A}_{2}$ is a coherent $\mathbb{A}^{1}$-flat sheaf on $X$, which is the direct sum of two line bundles on the generic fibre, but which is isomorphic to the indecomposable vector bundle $\mathcal{B}((1,-1), 1,-1)$ if restricted to the singular fibre $X_{0}$.

Example 4.3. In this example we benefit from the special choice of our family of cubics, which can be written in the form

$$
F_{t}(x, y, z)=\left(y^{2}-x^{2}\right) z-(x-t z)\left(x^{2}+t x z+t z^{2}\right) .
$$

We define $C \subset \mathbb{P}^{2} \times \mathbb{A}^{1}$ by the two equations

$$
x+y=0 \quad \text { and } \quad x^{2}+t x z+t z^{2}=0 .
$$

Clearly, $C \subset X$ and the support of $C$ is disjoint to the set given by $z=0$. Therefore, we restrict our attention to the affine open set $z \neq 0$, in which $C$ is given by the ideal

$$
I=\left\langle x+y, x^{2}+t x+t\right\rangle \subset \boldsymbol{k}[x, y, t] .
$$

For any $t \in \boldsymbol{k}$, the vector space $\boldsymbol{k}[x, y] / I$ is of dimension two. Moreover, $\boldsymbol{k}[x, y, t] / I$ is a flat $\boldsymbol{k}[t]$-module, because $t$ is not a zero-divisor of it. Hence, the sheaf $\mathcal{O}_{C}$ is a flat family of torsion sheaves of length two. For generic $t$, this module is supported at two distinct points whose coordinates $(x, y)=(x,-x)$ satisfy $x^{2}+t x+t=0$.

The fibre of $\mathcal{O}_{C}$ over $t=0$ is isomorphic to the module

$$
M=\boldsymbol{k}[x, y] /\left\langle x+y, x^{2}\right\rangle
$$


over the ring $R=\boldsymbol{k}[x, y] /\left\langle y^{2}-x^{2}-x^{3}\right\rangle$, and is supported at $(x, y)=$ $(0,0)$ only. Using $\widetilde{x}=x \sqrt{1+x} \in \widehat{R}$ and $\xi=\widetilde{x}-y, \eta=\widetilde{x}+y$ as before, we obtain $\widetilde{x}=x$ in $\widehat{M}$, the completion of $M$ at $(0,0)$. This implies $\eta=0$ and $\xi^{2}=4 \widetilde{x}^{2}=4 x^{2}=0$ in $\widehat{M}$, so that we obtain

$$
\widehat{M} \cong \widehat{R} /\left\langle\xi^{2}, \eta\right\rangle \text {. }
$$

This module is called a string module in the classification of Gelfand, Ponomarev and was denoted $\mathcal{N}(0() 1)$ in [12]. It has length two as expected. As in the previous example, we use the calculation from 12, which shows $\mathbb{F}(\mathcal{S}(0,-1)) \cong \mathcal{N}(0() 1)$, where $\mathcal{S}(0,-1)$ is a semistable torsion free coherent sheaf of rank two and degree zero on $X_{0}$. Therefore, we obtain

$$
\mathrm{FM}_{\mathcal{P}_{0}}^{\mathrm{b}}\left(i_{0}^{*} \mathcal{O}_{C}\right) \cong \mathcal{S}(0,-1)
$$

and $\mathrm{FM}_{\mathcal{P}}^{\mathrm{b}}\left(\mathcal{O}_{C}\right)$ is an $\mathbb{A}^{1}$-flat family of coherent sheaves on $X$ whose general fibre is the direct sum of two line bundles. The restriction to the singular fibre $X_{0}$, however, is indecomposable and not locally free.

\section{REFERENCES}

[1] A. Altman, S. Kleiman, Compactifying the Picard scheme, Adv. Math. 35 (1980) 50-112.

[2] A. Altman, S. Kleiman, Compactifying the Picard scheme II, Am. J. Math. 101 (1979) 10-41.

[3] B. Andreas, G. Curio, D. Hernández Ruipérez, S.-T. Yau, Fourier-Mukai Transform and Mirror Symmetry for D-Branes on Elliptic Calabi-Yau, arXiv: math.AG/0012196

[4] C. Bartocci, U. Bruzzo, D. Hernández Ruipérez, J. M. Muñoz Porras, Mirror symmetry on K3 surfaces via Fourier-Mukai transform, Comm. Math. Phys. 195 (1998) 79-93, arXiv: alg-geom/9704023

[5] D. Ben-Zvi, T. Nevins, From solitons to many-body systems, arXiv: math.AG/0310490

[6] A. Bondal, D. Orlov, Semiorthogonal decomposition for algebraic varieties, arXiv: alg-geom/9506012.

[7] T. Bridgeland, Fourier-Mukai transforms for elliptic surfaces, J. Reine Angew. Math. 498 (1998), 115-133.

[8] T. Bridgeland, Equivalences of triangulated categories and FourierMukai transforms, Bull. Lond. Math. Soc. 31 (1999) 25-34, arXiv: math.AG/9809114

[9] T. Bridgeland, Flops and derived categories, Invent. Math. 147, no. 3 (2002) 613-632, arXiv: math.AG/0009053

[10] T. Bridgeland, A. Maciocia, Fourier-Mukai transforms for $K 3$ and elliptic fibrations, J. Algebraic Geom. 11 no. 4 (2002) 629-657.

[11] W. Bruns, J. Herzog, Cohen-Macaulay Rings, Cambridge Studies in Advanced Mathematics 39 (1998). 
[12] I. Burban, B. Kreußler, Fourier-Mukai transforms and semi-stable sheaves on nodal Weierstrass cubics, J. Reine Angew. Math. (to appear), arXiv: math.AG/0401437

[13] A. Caldararu, Derived categories of twisted sheaves on elliptic threefolds, J. Reine Angew. Math. 544 (2002), 161-179.

[14] J.-C. Chen, Flops and equivalences of derived categories for threefolds with only terminal Gorenstein singularities, J. Differ. Geom. 61, no. 2 (2002) 227-261, arXiv: math.AG/0202005

[15] B. Conrad, Grothendieck Duality and Base Change, Lecture Notes in Mathematics 1750, Springer (2000).

[16] R. Donagi, T. Pantev, Torus fibrations, gerbes, and duality, arXiv: math.AG/0306213

[17] R. Friedman, J. Morgan, E. Witten, Vector bundles over elliptic fibrations, J. Algebr. Geom. 8, no. 2 (1999) 279-401.

[18] S. Gelfand, Y. Manin, Methods of Homological Algebra, Springer (2003).

[19] A. Grothendieck, Éléments de géométrie algébrique, Publ. Math., Inst. Hautes Étud. Sci. 4, 17, 20, 24, 28, 32, (1960-1967).

[20] R. Hartshorne, Residues and duality, Lecture Notes in Mathematics 20, Springer (1966).

[21] L. Illusie, Existance de résolutions globales, Exposé II, Thé orie des intersections et théorème de Riemann-Roch, Séminaire de Géométrie Algèbrique du Bois-Marie 1966-1967 (SGA 6), Lect. Notes Math. 225, (1971) 160-221.

[22] Y. Kawamata, D-equivalence and $K$-equivalence, J. Differ. Geom. 61, no. 1 (2002) 147-171, arXiv: math.AG/0205287.

[23] J. Lipman, Notes on Derived Categories and Derived Functors, available on http://www.math. purdue.edu/ lipman/.

[24] D. Mumford, Abelian Varieties, Tata Institute of Fundamental Research Studies in Mathematics 5, Oxford University Press (1970).

[25] S. Mukai, Duality between $D(X)$ and $D(\hat{X})$ with its application to Picard sheaves, Nagoya Math. J. 81 (1981) 153-175.

[26] D. O. Orlov, Derived categories of coherent sheaves and equivalences between them, Uspekhi Mat. Nauk 58, no. 3 (2003) 89-172; translation in Russian Math. Surveys 58, no. 3 (2003) 511-591.

[27] N. Spaltenstein, Resolutions of unbounded complexes, Compos. Math. 65, no. 2 (1988) 121-154.

[28] M. Van den Bergh, Three-dimensional flops and noncommutative rings, Duke Math. J. 122, no. 3 (2004) 423-455.

[29] C. Weibel, An introduction to homological Algebra, Cambridge Studies in Advanced Mathematics 38, Cambridge University Press (1994).

[30] K. Yoshioka, Moduli spaces of stable sheaves on abelian surfaces, Math. Ann. 321 no. 4 (2001) 817-884.

Max Planck-Institut für Mathematik, Vivatsgasse 7, 53111 Bonn, GERMANY

E-mail address: burban@mpim-bonn.mpg.de

Mary Immaculate College, South Circular Road, Limerick, Ireland

E-mail address: bernd.kreussler@mic.ul.ie 